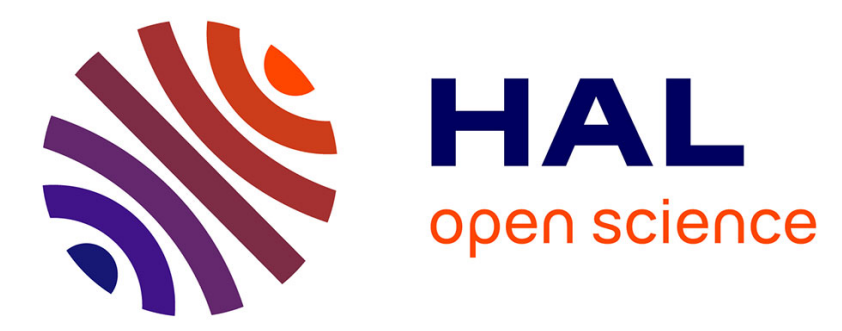

\title{
Uncovering the physiological and cellular effects of uranium on the root system of Arabidopsis thaliana
}

Nelson B.C. Serre, Claude Alban, Jacques Bourguignon, Stéphane Ravanel

\section{To cite this version:}

Nelson B.C. Serre, Claude Alban, Jacques Bourguignon, Stéphane Ravanel. Uncovering the physiological and cellular effects of uranium on the root system of Arabidopsis thaliana. Environmental and Experimental Botany, 2019, 157, pp.121-130. 10.1016/j.envexpbot.2018.10.004 . hal-02000621

\section{HAL Id: hal-02000621 \\ https://hal.univ-grenoble-alpes.fr/hal-02000621}

Submitted on 25 Sep 2020

HAL is a multi-disciplinary open access archive for the deposit and dissemination of scientific research documents, whether they are published or not. The documents may come from teaching and research institutions in France or abroad, or from public or private research centers.
L'archive ouverte pluridisciplinaire HAL, est destinée au dépôt et à la diffusion de documents scientifiques de niveau recherche, publiés ou non, émanant des établissements d'enseignement et de recherche français ou étrangers, des laboratoires publics ou privés. 


\section{Uncovering the physiological and cellular effects of uranium on the root system of Arabidopsis thaliana}

Nelson B. C. Serre, Claude Alban, Jacques Bourguignon* and Stéphane Ravanel*

Univ. Grenoble Alpes, CEA, CNRS, INRA, BIG-LPCV, 38000 Grenoble, France

${ }^{*}$ Authors for correspondence:

- Jacques Bourguignon, CEA, Univ. Grenoble Alpes, CNRS, INRA, BIG, PCV, 17 avenue des Martyrs, 38000 Grenoble, France. +33 438784688; jacques.bourguignon@cea.fr

- Stéphane Ravanel, INRA, Univ. Grenoble Alpes, CEA, CNRS, BIG, PCV, 17 avenue des Martyrs, 38000 Grenoble, France. +33 438783383; stephane.ravanel@cea.fr

\section{ABSTRACT}

Uranium $(\mathrm{U})$ is a naturally occurring radionuclide that is toxic for plants. The aim of this study was to gain insights into the physiological and cellular responses of roots to $U$ stress. We analyzed the effects of uranyl nitrate on the architecture and physiology of Arabidopsis thaliana roots using different staining procedures and reporter genes. Also, we examined the homeostasis of inorganic phosphate (Pi) and Fe during $U$ stress. We showed that, at a sub-toxic dose, $U$ stimulated the apex mitotic activity, resulting in improved primary root growth and reduced secondary root formation. At a toxic level, $U$ arrested primary root growth and increased the formation of secondary and higher-order lateral roots. $U$ stress was linked with a depletion of $\mathrm{Pi}$ and a redistribution of $\mathrm{Fe}$ in root tissues, together with the production of ROS and nitric oxide. Also, $U$ triggered the deposition of the defense polymers callose and lignin. These results showed that part of the radionuclide effects are linked with the signaling cascade of $\mathrm{Pi}$ sensing in the root apex. Other mechanisms involved in $U$ toxicity are likely related to perturbations of Fe homeostasis and direct deleterious effects of $U$ on root components.

\section{KEYWORDS}

Callose, iron, lignin, phosphate, root development, uranium. 


\section{INTRODUCTION}

Uranium $(U)$ is a non-essential trace metal element and radionuclide that is naturally present at $3 \mathrm{mg} / \mathrm{kg}$ on average in the Earth's crust (Anke et al., 2009). It is mainly redistributed in the environment by anthropogenic activities including $U$ and phosphate mining, nuclear industry, military activities, and soil fertilization (Vandenhove, 2002). Uranium may locally accumulate to concentrations that pose potential risks for ecosystems, agrosystems, and ultimately human health. Indeed, $U$ is chemotoxic and potentially radiotoxic (natural $U$ has a low specific activity) for all living organisms. Uranium is not essential for plants but it is taken up from the soil, incorporated into the biomass, and consequently enters the food chain. Thus, contamination of soils by $U$ and its absorption by plants represent a significant health risk for human beings (Anke et al., 2009; Schnug and Lottermoser, 2013). After ingestion, most $U$ is excreted within a few days and the small fraction $(0.2-5 \%)$ that is absorbed into the bloodstream is deposited preferentially in bone and kidneys, where it can cause diseases (e.g. Thiebault et al., 2007; Vidaud et al., 2012; Schnug and Lottermoser, 2013).

According to the hard and soft acid base Lewis concept, uranyl $\left(\mathrm{UO}_{2}{ }^{2+}\right)$ is a hard acid that reacts with hard bases such as phosphate, carbonate and hydroxyl groups (Pearson, 1963). This property, combined with other parameters such as the $\mathrm{pH}$ or the presence of organic acids, influences uranyl speciation in soil and water, its absorption by plants, and its interference with plant physiology and development (e.g. Ebbs et al., 1998; Vanhoudt et al., 2008; Misson et al., 2009; Laurette et al., 2012a,b). Uranium interferes with plant nutrition, photosynthesis, and induces an oxidative stress (e.g. Vanhoudt et al., 2011a,b,c, 2014; Aranjuelo et al., 2014; Saenen et al., 2014, 2015; Tewari et al., 2015). Also, it has been shown that $U$ perturbs iron and phosphate homeostasis and may lead to phosphate starvation (Misson et al., 2009; Doustaly et al., 2014; Berthet et al., 2018).

It is well known that the root system architecture is modulated in response to changes in nutrient availability and the presence of non-essential toxic metals in the rhizosphere (for a review, see Potters et al., 2009). The molecular and cellular events that trigger morphogenic responses of the root system have been described in details for several trace elements including copper (Lequeux et al., 2010), aluminum (Kopittke et al., 2015) or chromium (Eleftheriou et al., 2015), but not for U. Plants are able to integrate internal nutritional status together with external cues to activate the right modifications for the adaptation to a new environment. Messenger molecules are important for such responses (Corpas and Barroso, 2015; Mittler, 2017). For instance, reactive oxygen species (ROS) and nitric oxide (NO) are acting in synergy to induce the modulation of root growth and even trigger programmed cell death (Correa-Aragunde et al., 2004; Locato et al. 2016). Other actors of this response are hormones. Auxin plays an important role in plant development and is a major regulator of the root system architecture. At the root apex level it maintains the meristem activity and is also implicated in the initiation and development of lateral roots (De Smet et al., 2007; Petrasek and Friml, 2009; Laskowski and Ten Tusscher, 2017). Auxin is also the link between environmental signals and root development (Kazan, 2013).

Nevertheless, the effect of $U$ on root development and architecture is poorly described. It has been shown that $U$ can stimulate or inhibit the primary root growth as a function of its concentration and 
speciation (Misson et al., 2009). Also, $\mathrm{U}$ induces the accumulation of hydrogen peroxide and $\mathrm{NO}$ at the primary root apex (Tewari et al., 2015). These data are not sufficient per se to understand the cascade of cellular events that trigger changes in root development during $U$ stress. In order to fill this gap, we analyzed the impact of $U$ on the physiological and cellular processes that control root development and architecture. We showed that $U$ modifies primary and secondary root growth through the modulation of the meristem mitotic activity. Also, we could dissect for the first time the sequence of cellular mechanisms that are induced by $U$ in roots. These events comprise the induction of $\mathrm{Pi}$ deficiency, changes of Fe distribution in root tissues, the production of ROS and NO as messengers, and the accumulation of the cell wall polymers callose and lignin to control spreading of the toxic metal.

\section{MATERIALS AND METHODS}

\subsection{Plant growth conditions}

Wild-type Arabidopsis thaliana (ecotype Columbia, Col-0) and the following transgenic lines were used in this study: PIN2::PIN2-GFP (Blilou et al., 2005), DR5::GFP (Lequeux et al., 2010), and CYCB1::GUS (Lequeux et al., 2010). Seeds were surface sterilized and stored in water for 2 days at $4^{\circ} \mathrm{C}$ in the dark. After stratification, seeds were sown into homemade 3D printed hydroponic floating plates containing conical holes filled with $0.65 \%$ (w/v) agar (Agar plant type A, Sigma-Aldrich) solubilized in distilled water.

Plates were thermoprinted using the amorphous polymer acrylonitrile butadiene styrene using an Ultimaker 2 3D-printer (Ultimaker BV, The Netherlands). Plates were transferred to plastic containers filled with $200 \mathrm{~mL}$ of a nutrient solution composed of $0.88 \mathrm{mM} \mathrm{K}_{2} \mathrm{SO}_{4}, 2 \mathrm{mM} \mathrm{Ca}\left(\mathrm{NO}_{3}\right), 1 \mathrm{mM} \mathrm{MgSO}_{4}$, $0.25 \mathrm{mM} \mathrm{KH}_{2} \mathrm{PO}_{4}, 10 \mu \mathrm{M} \mathrm{H}_{3} \mathrm{BO}_{3}, 0.1 \mu \mathrm{M} \mathrm{CuSO}_{4}, 0.6 \mu \mathrm{M} \mathrm{MnSO}_{4}, 0.01 \mu \mathrm{M}\left(\mathrm{NH}_{4}\right)_{6} \mathrm{Mo}_{7} \mathrm{O}_{24}, 10 \mu \mathrm{M} \mathrm{ZnSO}$, $10 \mu \mathrm{M} \mathrm{NaCl}, 20 \mu \mathrm{M}$ Fe-EDTA, and 0.25 mM MES, pH 5.8 (Meyer et al., 2015). Seedlings were grown for 6 days in a growth chamber $\left(21^{\circ} \mathrm{C}\right.$, light intensity of $80 \mu \mathrm{mol}$ photons $\mathrm{m}^{-2} \mathrm{~s}^{-1}, 16 \mathrm{~h}$ of light per day). They were then transferred for up to 9 days onto a no phosphate (NP) or a low phosphate medium (LP, $\left.25 \mu \mathrm{M} \mathrm{KH} \mathrm{KO}_{4}\right)$ supplemented or not with 10 to $50 \mu \mathrm{M}$ of uranyl nitrate $\left(\mathrm{UO}_{2}\left(\mathrm{NO}_{3}\right)_{2}\right)$. Prior to any subsequent analysis, seedlings were rinsed once with a solution of $10 \mathrm{mM} \mathrm{Na}_{2} \mathrm{CO}_{3}$ and then twice with distilled water to remove uranyl adsorbed at the root surface (Doustaly et al., 2014).

\subsection{Analysis of root architecture}

Roots of freshly harvested seedlings were transferred into square Petri dishes filled with $0.5 \%(\mathrm{w} / \mathrm{v})$ agarose for scanning with a GS-800 scanner (BioRad). The primary root length and the number of secondary roots were measured with the ImageJ software.

\subsection{Cellular and molecular staining procedures}

Cell viability at the root apex was assessed using a dual staining with fluorescein diacetate (FDA) and propidium iodide (PI) (Jones et al., 2016). The staining solution was prepared by mixing FDA (Thermo Fisher Scientific) and PI (Sigma-Aldrich) commercial solutions in NP or LP medium to obtain final concentrations of $5 \mu \mathrm{g} / \mathrm{mL}$ FDA and $10 \mu \mathrm{g} / \mathrm{mL}$ PI. Seedlings were stained for $20 \mathrm{~min}$ in the dark and 
rinsed with NP or LP medium. FDA staining was observed with a FITC filter (excitation $480 \mathrm{~nm}$; emission $543 \mathrm{~nm}$ ) and PI with a Texas Red filter (excitation $580 \mathrm{~nm}$; emission $610 \mathrm{~nm}$ ). After acquisition, the green and red channels were fused with the Zen microscopy software (Zeiss).

Iron staining was achieved using the Perls method followed by a 3,3'-diaminobenzidine (DAB) intensification (Roschzttardtz et al., 2009). Seedlings were stained for $45 \mathrm{~min}$ in a $2 \%(\mathrm{v} / \mathrm{v}) \mathrm{HCl}$ and $2 \%$ $(\mathrm{w} / \mathrm{v}) \mathrm{K}_{4} \mathrm{Fe}(\mathrm{CN})_{6}$ solution and rinsed in water. For $\mathrm{DAB}$ intensification seedlings were transferred to a methanol solution containing $0.065 \%(\mathrm{w} / \mathrm{v}) \mathrm{NaN}_{3}$ and $0.3 \%(\mathrm{v} / \mathrm{v}) \mathrm{H}_{2} \mathrm{O}_{2}$ and incubated for 1 hour. Finally,

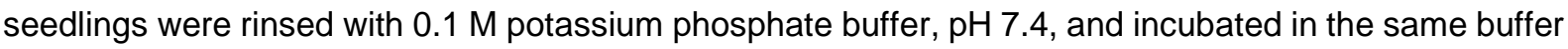
containing $0.00005 \%(\mathrm{w} / \mathrm{v}) \mathrm{CoCl}_{2}, 0.00002 \%(\mathrm{v} / \mathrm{v}) \mathrm{H}_{2} \mathrm{O}_{2}$ and $0.025 \%(\mathrm{w} / \mathrm{v}) \mathrm{DAB}$. The intensification was stopped after $5 \mathrm{~min}$ by rinsing seedlings with water.

ROS spatial and temporal distribution was observed using 2,7-dichlorofluorescein diacetate (Thermo Fisher Scientific) at the concentration of $2 \mu \mathrm{M}$ in NP or LP medium. Seedlings were stained for $20 \mathrm{~min}$ in the dark and then rinsed in NP or LP medium. Stained seedlings were observed with a FITC filter with the same exposure time of $120 \mathrm{~ms}$.

Nitric oxide accumulation was followed with 4,5-diaminofluorescein diacetate (Abcam) prepared in DMSO and diluted to a final concentration of $10 \mu \mathrm{M}$ in the staining solution (20 mM HEPES, pH 7.2). Seedlings were stained for $10 \mathrm{~min}$ in the dark and then rinsed in the HEPES buffer. Stained seedlings were observed with a FITC filter with the same exposure time of $400 \mathrm{~ms}$.

Callose staining was performed according to the method of Schenk and Schikora (2016). Seedlings were rinsed with a solution of $150 \mathrm{mM} \mathrm{KH}_{2} \mathrm{PO}_{4}$, stained for $25 \mathrm{~min}$ in the dark in a staining solution containing $0.01 \%(\mathrm{w} / \mathrm{v})$ aniline blue and $150 \mathrm{mM} \mathrm{KH}_{2} \mathrm{PO}_{4}$, and then rinsed with a solution of $150 \mathrm{mM}$ $\mathrm{KH}_{2} \mathrm{PO}_{4}$. Stained seedlings were observed with a DAPI filter (excitation $365 \mathrm{~nm}$; emission: $460 \mathrm{~nm}$ ) with the same exposure time of $200 \mathrm{~ms}$.

Lignin, or Wiesner, staining was performed according to Pradhan Mitra and Loqué (2014). A 3\% (w/v) solution of phloroglucinol (Prolabo) freshly prepared in ethanol was mixed with $37 \%(\mathrm{w} / \mathrm{v})$ hydrochloric acid in a ratio of 2:1. Seedlings were directly stained on a microscope slide with the Wiesner solution for 5 min and observed under differential interference contrast light.

For $\beta$-glucuronidase activity staining seedlings were fixed in cold $90 \%(\mathrm{v} / \mathrm{v})$ acetone for $20 \mathrm{~min}$ and incubated overnight at $37^{\circ} \mathrm{C}$ in a GUS staining solution containing $0.2 \%(\mathrm{v} / \mathrm{v})$ Triton X-100, $50 \mathrm{mM}$ $\mathrm{NaH}_{2} \mathrm{PO}_{4} / \mathrm{Na}_{2} \mathrm{HPO}_{4}$, pH 7.2, 2 mM potassium ferrocyanide, $2 \mathrm{mM}$ 5-Bromo-4-chloro-1H-indol-3-yl $\beta$-Dglucopyranosiduronic acid. After 24 hours of incubation, seedlings were observed under differential interference contrast light.

Stained seedlings or root sections were mounted onto microscope slides in $10 \%(\mathrm{v} / \mathrm{v})$ glycerol and observed under a Leica Axioscope A1 microscope with the suitable filter.

\subsection{Optical section fluorescence microscopy}

Structured illumination for optical sectioning fluorescence microscopy has been conducted for the observation of DR5::GFP and PIN2::PIN2-GFP seedlings using an Axioimager Z3 Apotome microscope (Zeiss). The DR5::GFP signal was observed under a GFP filter with the same time of exposure of 120 ms. 


\subsection{Determination of $U$ by inductively coupled plasma mass spectrometry (ICP-MS)}

Seedlings were harvested after 3 days of treatment and digested at $90^{\circ} \mathrm{C}$ for 4 hours in $65 \%(\mathrm{w} / \mathrm{v}) \mathrm{HNO}_{3}$. Mineralized samples were diluted in $0.5 \%(\mathrm{v} / \mathrm{v}) \mathrm{HNO}_{3}$ and analyzed using an iCAP RQ quadrupole mass instrument (Thermo Fisher Scientific $\mathrm{GmbH}$, Germany). ${ }^{238 \mathrm{U}}$ concentration was determined using a standard curve and corrected using an internal standard solution of ${ }^{172} Y$ added online. Data integration was done using the Qtegra software (Thermo Fisher Scientific GmbH, Germany).

\subsection{Quantification of inorganic phosphate}

Seedlings treated for 3 days were harvested, frozen in liquid nitrogen, and grinded into a fine powder. Soluble inorganic phosphate (Pi) was extracted using the protocol of Kanno et al. (2016). Samples were suspended in a buffer containing $170 \mathrm{mM}$ MES pH 5.8 and $100 \mathrm{mM}$ dithiothreitol (10 $\mu \mathrm{L}$ of buffer per $\mathrm{mg}$ of powder) and incubated for $10 \mathrm{~min}$ at $4^{\circ} \mathrm{C}$. After centrifugation, $20 \mu \mathrm{L}$ of the supernatant was mixed with an acidic molybdate solution (1.755\% (w/v) $\left(\mathrm{NH}_{4}\right)_{6} \mathrm{Mo}_{7} 4 \mathrm{H}_{2} \mathrm{O}$ in $\left.3 \mathrm{M} \mathrm{H}_{2} \mathrm{SO}_{4}\right)$. After $10 \mathrm{~min}$ of incubation, the malachite green solution containing $0.35 \%(\mathrm{w} / \mathrm{v})$ polyvinylic alcohol and $0.035 \%(\mathrm{w} / \mathrm{v})$ malachite green was added. Samples were incubated for 2 hours and the absorption measured at 610 $\mathrm{nm}$ with a Tecan Spark plate reader. Pi contents were determined using a calibration curve made with a solution of $\mathrm{KH}_{2} \mathrm{PO}_{4}$.

\subsection{Statistical analysis}

Statistical analysis was conducted using the $R$ software (R Core Team, 2017) and the nparcomp package (Konietschke et al., 2015). Each parameter was compared to the control LP medium with a non-parametric Dunnett test coupled with a Fisher asymptotic approximation method. Confidence level was set at $95 \%(p$-value $<0.05)$. 


\section{RESULTS}

\subsection{Uranium affects cell viability in the primary root apex of Arabidopsis}

We developed an experimental setup to analyze the physiological and cellular effects of $U$ on the root system architecture of $A$. thaliana. The toxicity of metals on plant roots is frequently analyzed using agar-solidified culture media on which seedlings are grown in a vertical orientation (e.g. Howden and Cobbett, 1992; Hugouvieux et al., 2009; Lequeux et al., 2010). We first used this procedure to determine which concentrations of uranyl nitrate induce significant effects on the growth and architecture of primary roots of $A$. thaliana Col- 0 seedlings. It turned out that a high concentration of $U$ (above $100 \mu \mathrm{M}$ ) was necessary to significantly inhibit root growth of 4-day-old seedlings in these conditions (data not shown). This low sensitivity was likely due to a low availability of $U$ because of strong interactions of uranyl ions with the hydroxyl moieties of the agar molecule and with traces of inorganic phosphate $(\mathrm{Pi})$ that are present in this gelling agent (Misson et al., 2009; Jain et al., 2009). Then, we designed an alternative experimental setup using hydroponics for a better control of $U$ bioavailability. In this system, Col-0 seeds were germinated and grown for 6 days in a hydroponic culture medium containing sufficient amounts of all nutrients, including $250 \mu \mathrm{M} \mathrm{Pi}$ (see Methods for details). At this stage the primary root was about 0.5 $\mathrm{cm}$ long and suitable to analyze the effects of $U$ stress. Since $P i$ is a ligand of $U, 6$-day-old seedlings were transferred onto a medium with a low Pi content ( $25 \mu \mathrm{M} \mathrm{Pi}$, LP medium) and treated with uranyl nitrate concentrations ranging from 0 to $50 \mu \mathrm{M}$. Seedlings were also analyzed in a medium containing no $\mathrm{Pi}$ at all (NP medium). We assessed $\mathrm{U}$ toxicity by following cell viability over time using a dual staining procedure in which living cells were stained in green with fluorescein diacetate and dead cells were stained in red with propidium iodide. No modification in cell viability was observed in NP, LP and LP plus $10 \mu \mathrm{M}$ uranyl nitrate over the 9 days of the experiment (Fig. 1). At $20 \mu \mathrm{M} \mathrm{U}$ (U20 condition), a weak toxicity was observed during the first two days of the treatment, with mainly living cells and some dead cells (Fig. 1). After 9 days in U20 the cell viability was similar to control apices but the root apex was swollen, as in NP, as compared to the LP medium. A strong cellular toxicity of the radionuclide was observed at $30 \mu \mathrm{M}$ of uranyl nitrate (U30 condition) and above (Fig. 1). In these conditions, cell viability started to decrease within the first 24 hours of $U$ stress, with significant cell death in the outermost cell layers (epidermis and cortex) of the meristematic and elongation zones (see Supplemental Fig. S1 for the organization of the root apex in the different conditions). At days 2 and 3 in U30, cell death spread to the whole primary root apex and abnormal lateral roots emerged in the differentiation zone (Fig. 1). Secondary roots were able to elongate in U30 but higher concentrations of uranyl nitrate fully abolished their growth. Given these results, we selected the $\mathrm{U} 20$ and $\mathrm{U} 30$ conditions as sub-toxic and toxic doses of uranyl nitrate, respectively, to characterize the effects of the radionuclide on the root system of Arabidopsis. 


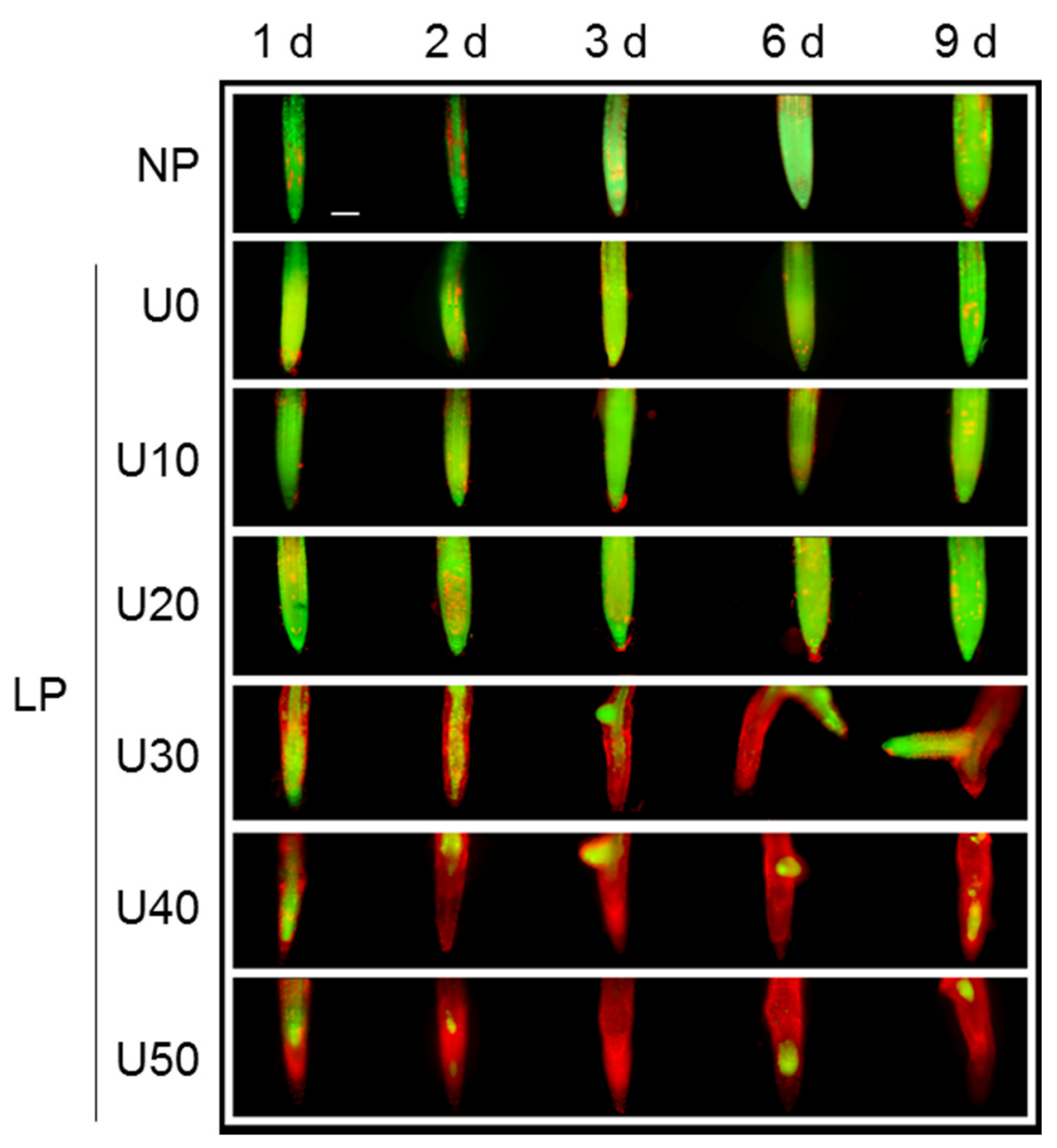

Figure 1. Uranium affects cellular viability in the primary root apex of Arabidopsis. A. thaliana Col-0 seeds were germinated and grown for 6 days in hydroponics with a complete nutrient solution. Seedlings where then transferred to either No Phosphate medium (NP) or Low Phosphate medium (LP, $25 \mu \mathrm{M} \mathrm{Pi}$ ) containing 0 to $50 \mu \mathrm{M}$ of uranyl nitrate (U0-U50). Cell viability of the primary root apex was monitored after 1, 2, 3, 6 and 9 days of treatment. Living cells were stained in green with fluorescein diacetate and dead cells in red with propidium iodide. Observations were made at magnification x200 using an epifluorescence microscope. Fluorescein diacetate and propidium iodide were observed under FITC and Texas Red filters, respectively. Images are representative of $n=8$ samples analyzed at each time-point in two independent experiments. Scale bar $=100 \mu \mathrm{m}$.

\subsection{Uranium perturbs phosphate and iron homeostasis in the root}

It has been reported that $\mathrm{U}$ interferes with $\mathrm{Pi}$ and Fe homeostasis at the root and shoot levels (Vanhoudt et al., 2011a; Doustaly et al., 2014; Berthet et al., 2018) and that Pi has a central role in U immobilization in root tissues (Misson et al., 2009; Laurette et al., 2012a). To have a better understanding of $U$ toxicity in our experimental conditions we measured $\mathrm{U}, \mathrm{Pi}$ and $\mathrm{Fe}$. $\mathrm{U}$ was determined by ICP-MS following sample mineralization in concentrated nitric acid, whereas $\mathrm{Pi}$ was measured spectrophotometrically using the molybdate/malachite green reagents following extraction of soluble compounds (Kanno et al., 2016). Seedlings treated with $30 \mu \mathrm{MU}$ for 3 days accumulated twice more of the toxic metal than seedlings treated with $20 \mu \mathrm{MU}$ (Fig. 2A). This significant difference could explain the important changes in root apex cell viability observed between U20 and U30 conditions (Fig. 1). Measurements of soluble $\mathrm{Pi}$ indicated that $\mathrm{U}$ stress at 20 or $30 \mu \mathrm{M}$ uranyl nitrate induced a Pi starvation in seedlings similar to the 
one measured in the NP condition. In these three conditions, the soluble Pi content was decreased by 55 to $65 \%$ as compared to the control LP medium (Fig. $2 \mathrm{~A}$ ).
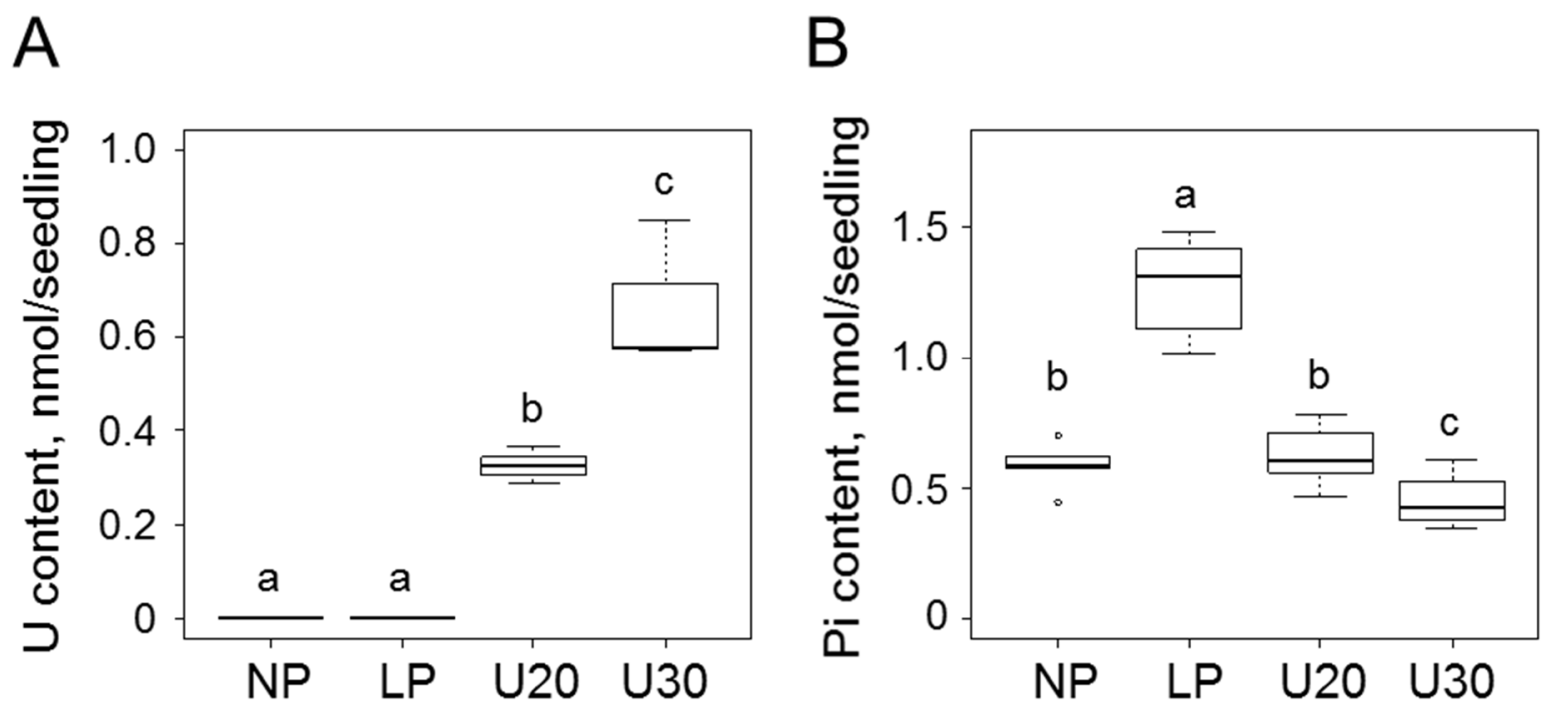

Figure 2. Uranium disrupts phosphate homeostasis in Arabidopsis seedlings. Six-day-old Col-0 seedlings grown in Pi-sufficient conditions were transferred to either No Phosphate medium (NP) or Low Phosphate medium (LP, $25 \mu \mathrm{M} \mathrm{Pi}$ ) containing 20 or $30 \mu \mathrm{M}$ uranyl nitrate (U20, U30) for 3 days. A- The amount of $U$ in seedlings was determined by ICP-MS following sample mineralization in concentrated nitric acid. $\mathrm{B}$ - The amount of soluble $\mathrm{Pi}$ in seedlings was measured spectrophotometrically using the molybdate/malachite green reagents following extraction of soluble compounds in a MES/DTT buffer (Kanno et al., 2016). The distribution of data is displayed in Tukey's boxplots with the median as the solid line inside the box, the first and third quartiles as the bottom and top lines of the box, and whiskers with maximum 1.5 interquartile range of the lower and upper quartile, respectively. Outliers are plotted as individual dots. Each distribution represents $n=3-6$ pools of 8 seedlings. Statistical significance was determined using multiple nonparametric comparisons with Dunnett's test with $p<0.05$. Letters indicate significant differences.

Since Pi starvation was not proportional to $U$ content in the seedlings, we measured the concentration of both elements in the culture medium. Aliquots were taken at the surface of the medium, which corresponds to the zone of mineral root absorption. We observed that both $\mathrm{Pi}$ and $\mathrm{U}$ concentrations decreased rapidly and simultaneously during the first 3 days of the experiment (Supplemental Fig S2). After one day, the amount of $\mathrm{Pi}$ and $\mathrm{U}$ decreased by $60-70 \%$ in the root absorption zone, regardless of the initial concentration of uranyl nitrate. At day 3 , there was less than $5 \mu \mathrm{M} \mathrm{Pi}$ available for seedlings and the concentration of $U$ was about $2 \mu \mathrm{M}$ and $6 \mu \mathrm{M}$ in $\mathrm{U} 20$ and $\mathrm{U} 30$ conditions, respectively (Fig S2). These results most probably correspond to the formation of U-Pi complexes in the medium and their subsequent precipitation. Also, the similar extent of Pi depletion measured in the U20 and U30 media could explain that $\mathrm{Pi}$ starvation in the corresponding seedlings was comparable (Fig. 2B) and not proportional to the amount of absorbed $U$ (Fig. 2A).

We used the Perls-diaminobenzidine (DAB) histological staining procedure (Roschzttardtz et al., 2009) to investigate the effect of $U$ on the distribution of labile (non-heme) $\mathrm{Fe}^{2+} / \mathrm{Fe}^{3+}$ in the root apex. We observed similar patterns in the NP and U20 conditions with the accumulation of $\mathrm{Fe}$ in the stem cell niche and the elongation zone (Fig. 3). In the U30 condition, the distribution of Fe was markedly modified with a very strong Perls-DAB staining observed at the primary root apex and along the entire root axis 
(Fig. 3). Together these data showed that the $U 20$ and $U 30$ conditions lead to very different distributions of labile $\mathrm{Fe}$ in the root.

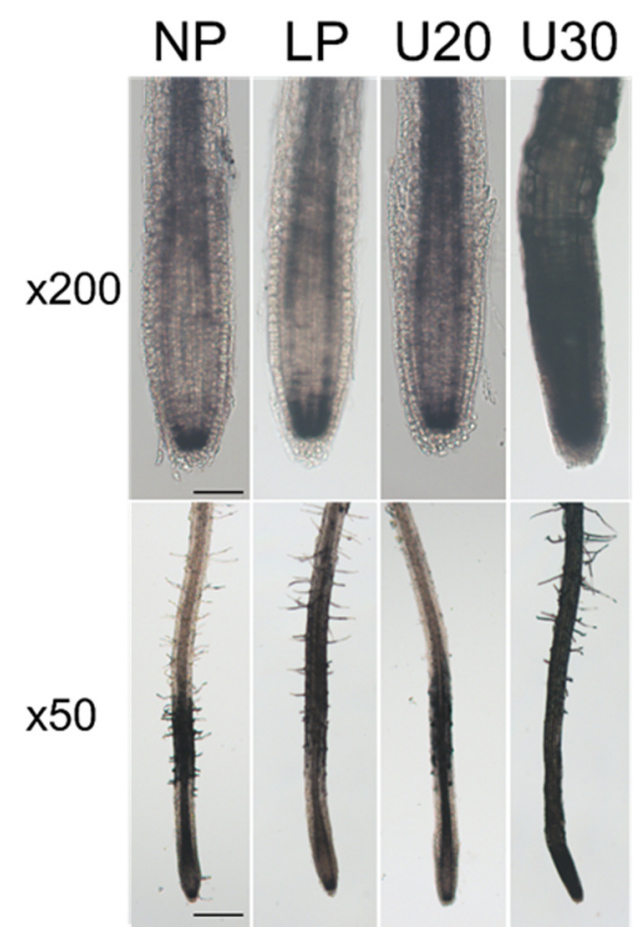

Figure 3. Uranium induces Fe redistribution in the root of Arabidopsis. Six-day-old Col-0 seedlings grown in Pi-sufficient conditions were transferred to either No Phosphate medium (NP) or Low Phosphate medium (LP, $25 \mu \mathrm{M} \mathrm{Pi}$ ) containing 20 or $30 \mu \mathrm{M}$ uranyl nitrate (U20, U30) for 3 days. The distribution of labile $\mathrm{Fe}^{2+} / \mathrm{Fe}^{3+}$ in roots was analyzed using the Perls-DAB staining. Observation of the dark-brown coloration typical of the Perls stain and DAB intensification (Roschzttardtz et al., 2009) was made at magnification $\times 200$ and $\times 50$ with an optical microscope under bright field. Images are representative of $n=4$ samples analyzed at each time-point in two independent experiments. Scale bar $=50 \mu \mathrm{m}$ for $\times 200$ and $200 \mu \mathrm{m}$ for $\times 50$.

\subsection{Uranium modifies the root architecture}

To analyze the effect of $U$ on the root architecture we measured the primary root length and the number of secondary roots during 9 days after the transfer of 6-day-old Pi-replete seedlings to challenging media. In the NP medium, the primary root length was significantly decreased as compared to the LP condition and the difference was observed since the first day of treatment (Fig. 4AB). Also, the number of lateral roots measured at day 9 was significantly higher in NP than in the LP condition (Fig. 4C). These results were expected since the typical response to Pi starvation is a decreased primary root growth and an increased lateral root development (Péret et al., 2011). At $20 \mu \mathrm{M}$ uranyl, the primary root length was not different from the control LP condition during the first 3 days of treatment. However, significant changes were observed at days 6 and 9 with longer primary roots in the U20 condition (Fig. $4 A B)$. Also, the number of secondary roots was significantly reduced in U20 as compared to the LP condition (Fig. 4C). At $30 \mu \mathrm{M}$ uranyl, the primary root growth was completely inhibited in the first 24 hours of treatment and we could not observe any reversibility of the inhibition over time (Fig. 4AB), even when $\mathrm{U}$ and Pi were markedly reduced in the culture medium (Fig S2). In the U30 condition, the number of secondary roots was significantly higher than in LP medium and comparable to the one measured in 
NP (Fig. 4C). However, there was a marked difference between these two conditions since secondary roots elongated in NP whereas they were rapidly arrested in U30 (Fig. 4A). Together, these data showed that the changes of the root architecture induced by uranyl at sub-toxic or toxic doses are not similar to the one induced by Pi deprivation on its own.
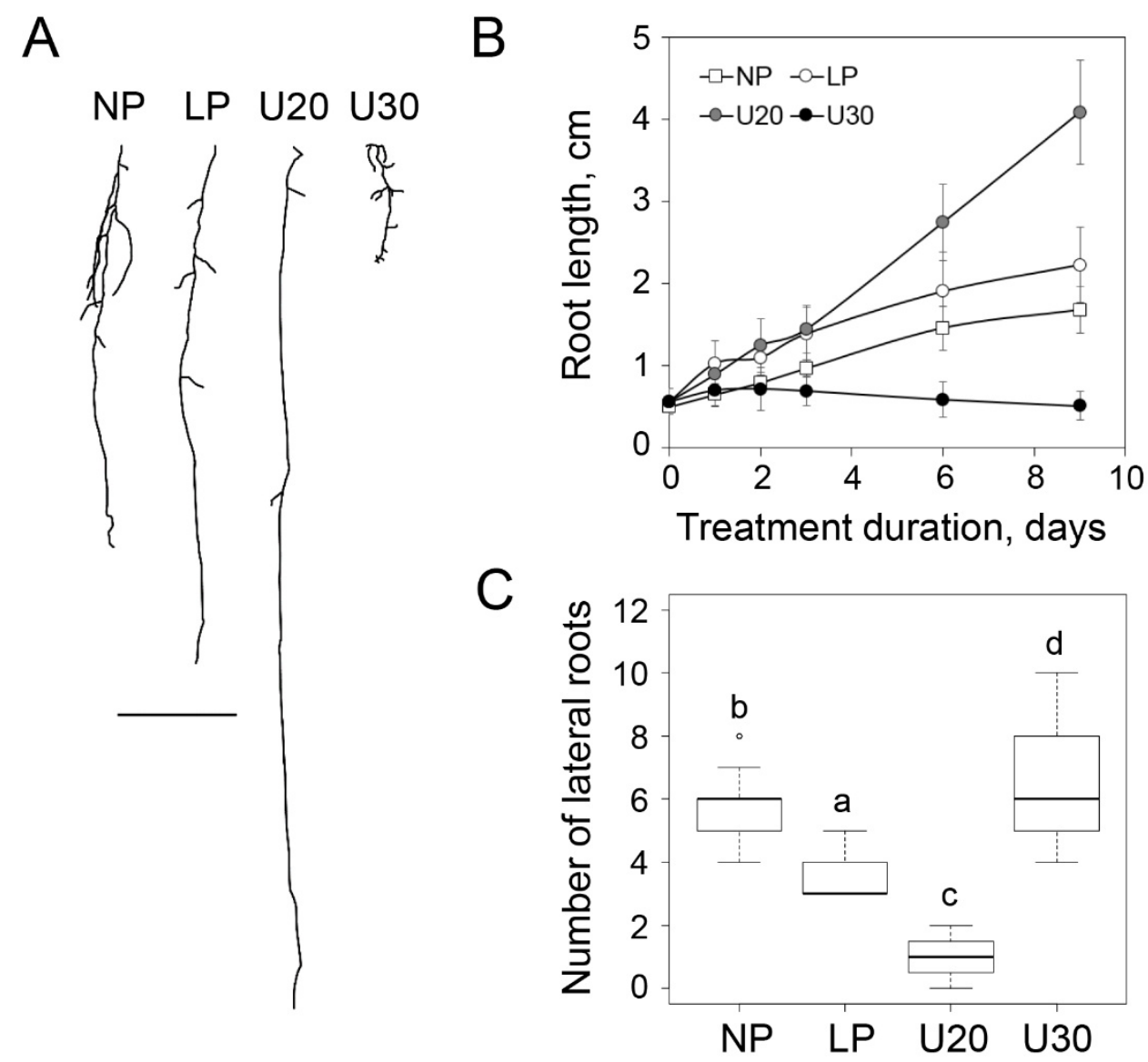

Figure 4. Uranium modulates the root architecture of Arabidopsis. Six-day-old Col-0 seedlings grown in Pi-sufficient conditions were transferred to either No Phosphate medium (NP) or Low Phosphate medium (LP, $25 \mu \mathrm{M}$ Pi) containing 20 or $30 \mu \mathrm{M}$ uranyl nitrate (U20, U30). A- Graphical representation of the typical root architecture observed after 9 days of treatment. Scale bar $=0.5 \mathrm{~cm}$. BKinetic of primary root growth. Primary root length was measured at regular time intervals. Curves represent mean $\pm S D$ with $n=20$ to 60 seedlings per condition. Root length in NP and U30 was significantly different from LP from days 1 to 9 ; for U20, significant difference was observed at days 6 and 9. C- Production of lateral roots. The number of lateral roots per seedling was counted after 9 days of treatment. Each distribution represents $n=12$ to 35 seedlings. Statistical significance was determined using multiple nonparametric comparisons with Dunnett's test with $p<0.05$. Letters indicate significant differences.

\subsection{Uranium modulates the mitotic activity in the root apex}

To gain insight into the mechanisms by which $U$ modifies primary root growth and lateral root initiation we used an Arabidopsis CYCB1::GUS reporter line. Cyclin B1 is essential for the G2/M cell cycle transition and its expression gives clues about the relative mitotic activity (Doonan, 1996). The activity of $\beta$-glucuronidase (GUS) was detected in the primary root apex in the control LP condition and it was less important in NP (Fig. 5). This is in agreement with the reduced root growth observed in the NP condition (Fig. 4B) and the reported consequence of Pi starvation (Svistoonoff et al., 2007). At $20 \mu \mathrm{M}$ 
uranyl, the mitotic activity was markedly increased during the first day of treatment and was maintained at a very high level till the end of the experiment (Fig. 5). In U30, GUS staining was also increased in the primary root apex during the first day of $U$ stress, but this situation was transient since no more activity could be detected beyond day 2 (Fig. 5). At these stages the CYCB1::GUS reporter was detected in lateral roots that were initiated close to the primary root apex. An Arabidopsis line expressing a 35S::GUS construct was used as a control. Uranyl nitrate at 20 or $30 \mu \mathrm{M}$ had no effect on GUS activity in this line (data not shown), indicating that the results we obtained are specific to the activity of the CYCB1 promoter. Together, these data indicated that changes in the root architecture under $U$ stress are related to perturbations of the mitotic activity in the primary and secondary root apices.

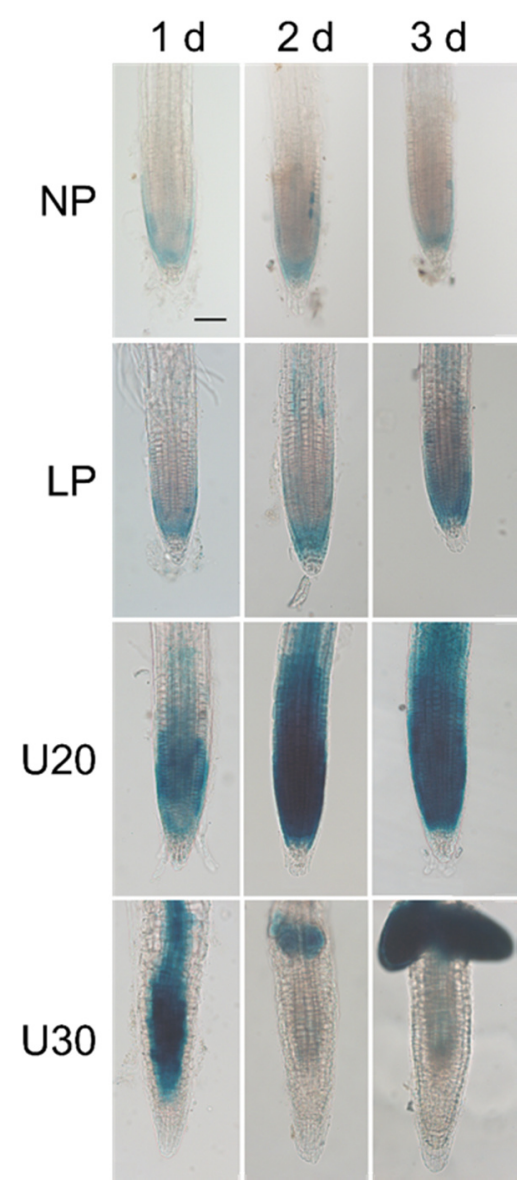

Figure 5. Uranium modulates the mitotic activity in the root apex of Arabidopsis. A. thaliana Col0 seeds expressing a CYCB1::GUS fusion were germinated and grown for 6 days in hydroponics with a complete nutrient solution. Seedlings where then transferred to either No Phosphate medium (NP) or Low Phosphate medium (LP, $25 \mu \mathrm{M}$ Pi) containing 20 or $30 \mu \mathrm{M}$ uranyl nitrate (U20, U30). GUS activity in root apices was observed after 1,2 and 3 days of treatment. Observations were made at magnification x200 under an optical microscope under bright light. Images are representative of $n=10$ samples analyzed at each time-point in two independent experiments. Scale bar $=100 \mu \mathrm{m}$.

\subsection{Uranium disrupts the transport and gradient of auxin in the root}

Auxin is known to play a major role in the maintenance of the meristem activity and the development of lateral roots (De Smet et al., 2007; Petrasek and Friml, 2009; Laskowski and Ten Tusscher, 2017). We analyzed auxin distribution in the root apex under $U$ stress using Arabidopsis seedlings expressing the 
auxin-reporter DR5::GFP construct. In LP, NP and U20 conditions, the pattern of DR5::GFP expression was similar and restricted to the stem cell niche and root cap (Fig. 6A). The activity of the DR5 promoter was less important in the U30 condition, with a decreased GFP signal after 1 day of treatment and no more detectable signal after 2 days of treatment (Fig. 6A).

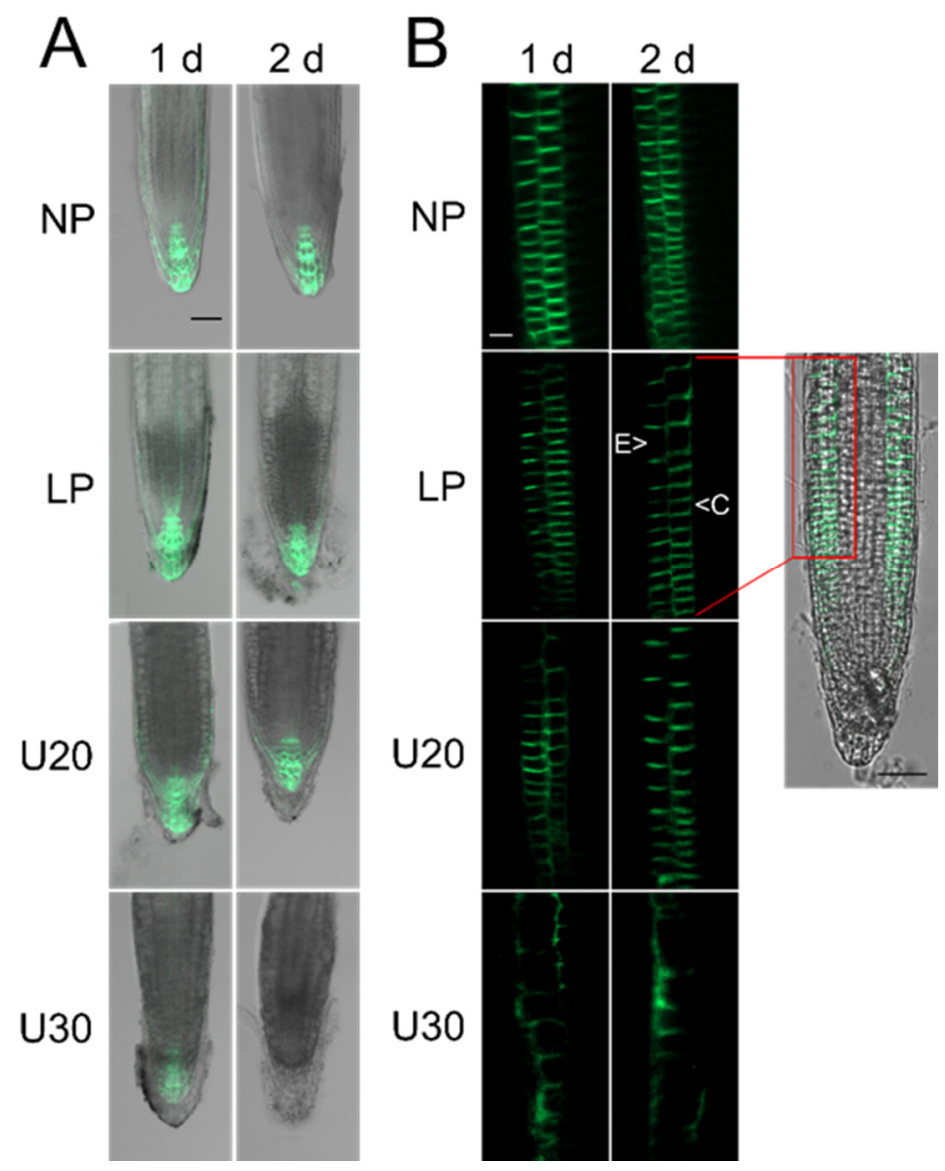

Figure 6. A toxic dose of uranium disrupts auxin homeostasis in the primary root apex of Arabidopsis. A. thaliana lines expressing either DR5::GFP or PIN2::PIN2-GFP were germinated and grown for 6 days in hydroponics with a complete nutrient solution. Seedlings where then transferred to either No Phosphate medium (NP) or Low Phosphate medium (LP, $25 \mu \mathrm{M}$ Pi) containing 20 or $30 \mu \mathrm{M}$ uranyl nitrate (U20, U30) for one or two days before observation. A- Expression of DR5::GFP in the root apex. The auxin-responsive DR5::GFP reporter is an indicator of auxin distribution. B- Expression of PIN2::PIN2-GFP in the root apex. PIN2 is the main auxin efflux transporter at the root apex, making the hormone returning toward the shoot. Epidermis $(\mathrm{E})$ and cortex $(\mathrm{C})$ cells are indicated by arrow heads. Optical sections were observed with an Axioimager Z3 Apotome microscope (Zeiss) at magnification x200 under a GFP filter and/or in bright field. Images in panel B were obtained with a digital zoom of 2.6. Images are representative of $n=6$ to 10 samples analyzed at each time-point in two independent experiments. Scale bar is $100 \mu \mathrm{m}$ in A, $50 \mu \mathrm{m}$ in B.

To study the inverted fountain mechanism that controls auxin distribution in the root apex we analyzed the expression of PIN2, one of the main transporters involved in this process (Petrasek and Friml, 2009). To this aim Arabidopsis seedlings expressing PIN2::PIN2-GFP were challenged with uranyl nitrate. The PIN2 transporter is normally present in the epidermis and cortex cells and this pattern was found in the LP, NP and U20 conditions (Fig. 6B). In the U30 condition, the pattern of PIN2-GFP expression was markedly modified with diffuse and less intense fluorescence signals after one day of treatment (Fig. 
6B). Together these data indicated that toxic levels of uranyl disrupt the transport and gradient of auxin in the root apex.

\subsection{Uranium induces the accumulation of ROS and the synthesis of NO in the root}

Uranium stress is known to induce the production of reactive oxygen species (ROS) and, hence, to trigger antioxidant defense mechanisms in plants (e.g. Vanhoudt et al., 2008; Vanhoudt et al., 2011bc; Aranjuelo et al., 2014). Also, it has been shown that the production of hydrogen peroxide and nitric oxide (NO) is linked with the toxicity of the radionuclide in roots and leaves (Tewari et al., 2015). To investigate the pattern of ROS production in the primary root of young Arabidopsis seedlings challenged with $U$, we used the fluorogenic reagent $2^{\prime}, 7^{\prime}$-dichlorodihydrofluorescein diacetate that produces a green fluorescence when oxidized in the cytosol. Our results showed that, while no specific fluorescence was observed in the LP and NP conditions, ROS were detected in primary root apices exposed to U (Fig. $7 \mathrm{~A})$. In the U20 condition, a small and transient increase in fluorescence was observed in the elongation zone after 24 hours of treatment. In the U30 condition, a strong oxidative burst was observed after 6 hours of treatment, mainly in the elongation zone (Fig. 7A). Then, the production of ROS spread to the entire root tip after 24 hours of treatment and, finally, the fluorescence signal decreased to background level after 3 days of stress.

We analyzed NO content in the primary root of Arabidopsis during $U$ stress using the green fluorescent indicator 4,5-diaminofluorescein diacetate (Fig. 7B). We showed that in the LP, NP and U20 conditions there was no noticeable change in fluorescence, while the U30 treatment triggered a strong production of NO. Indeed, a strong fluorescence was detected in the entire root tip after 6 hours of $U$ stress, whereas punctuated and dense signals were observed in the elongation zone at 24 hours, before the signal decreased to the background level at 72 hours (Fig. 7B). These results suggested that NO acts as a messenger molecule in roots exposed to a toxic dose of $U$. 


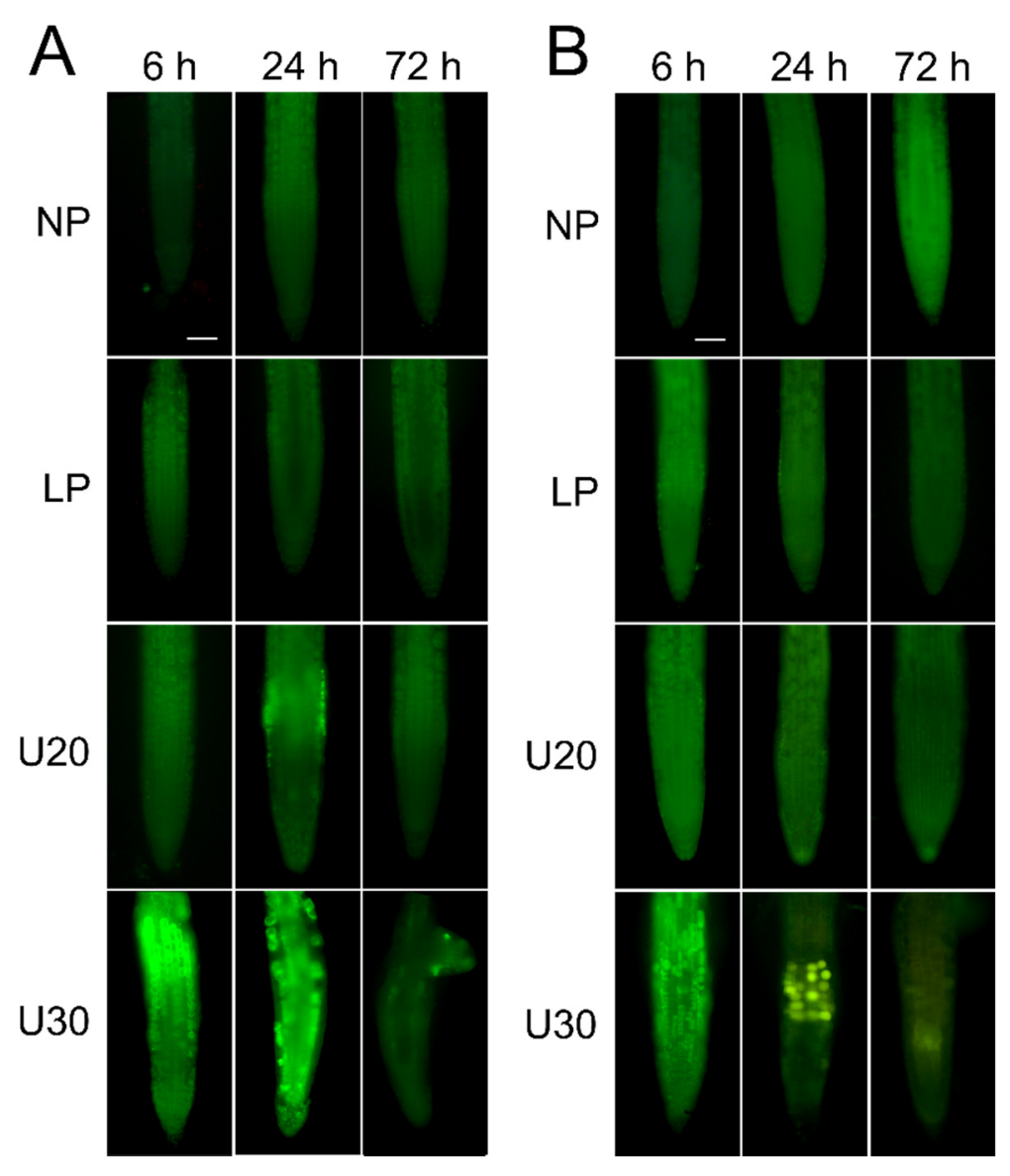

Figure 7. Uranium induces the production of ROS and NO in the primary root apex of Arabidopsis. Six-day-old seedlings grown in Pi-sufficient conditions were transferred to either No Phosphate medium (NP) or Low Phosphate medium (LP, $25 \mu \mathrm{M}$ Pi) containing 20 or $30 \mu \mathrm{M}$ uranyl nitrate (U20, U30) for 6 to 72 hours. A- Detection of ROS using 2',7'-dichlorodihydrofluorescein diacetate. BDetection of NO using 4,5-diaminofluorescein diacetate. Observation of both fluorescent reporters was done at magnification $\times 200$ with an epifluorescence microscope using a FITC filter. Exposure times were set at $120 \mathrm{~ms}(A)$ and $400 \mathrm{~ms}(B)$. Images are representative of $n=4$ to 20 samples analyzed at each time-point in two to four independent experiments. Scale bar $=100 \mu \mathrm{m}$.

\subsection{Uranium induces the accumulation of defense polymers in the root}

Callose is a polymer that controls the aperture of plasmodesmata and, thus, regulates symplasmic exchanges between plant cells (for a review, see Nedukha, 2015). The presence of callose at the root apex during $U$ stress was investigated using aniline blue staining. Callose production was detected specifically in response to $U$, with no fluorescent signal in the LP and NP conditions (Fig. 8A). In the U20 condition, callose deposition was more important in the elongation zone after one day of exposure to $U$ than after three days (Fig. 8A). In the U30 condition, the signal at day 1 was stronger than in U20 and enclosed the whole primary root apex with strong callose deposition in the meristematic zone (Fig. 8A). The fluorescent signal then disappeared after 3 days of treatment, making the callose accumulation/degradation spatiotemporal pattern similar to the one observed for ROS accumulation (Fig. 7A). 


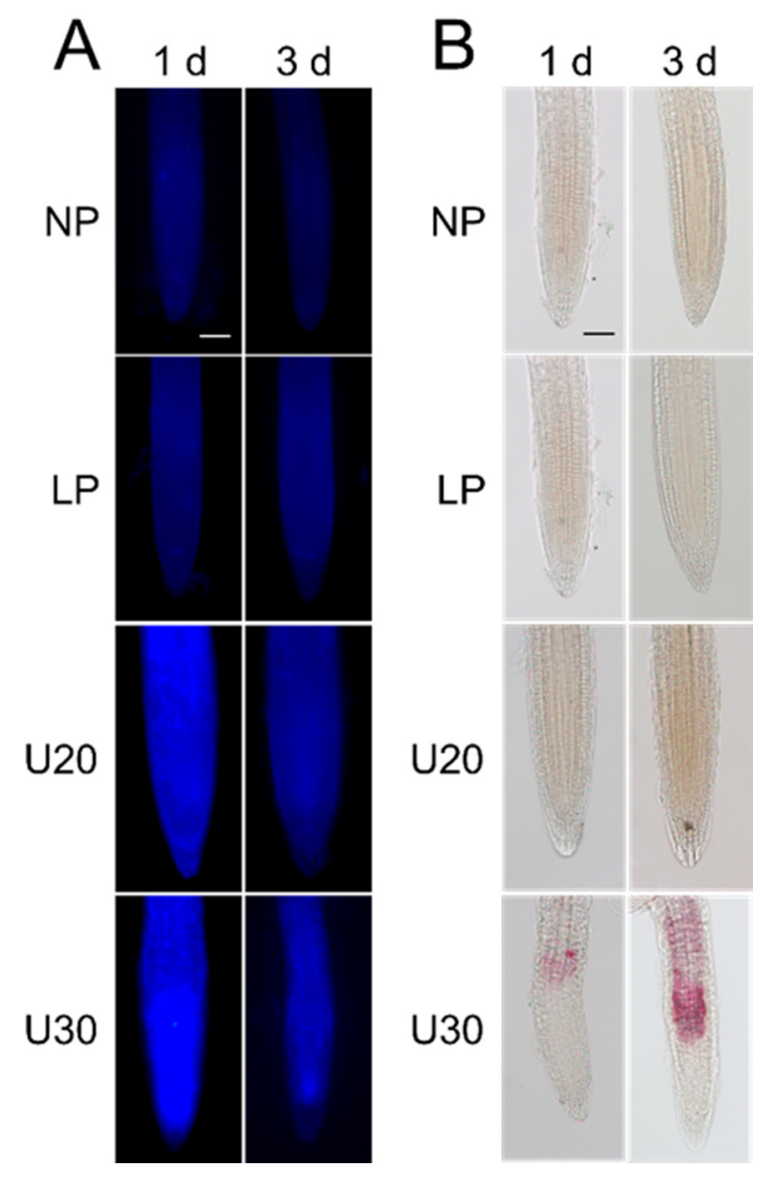

Figure 8. Uranium induces the synthesis and accumulation of defense polymers in the primary root of Arabidopsis. Six-day-old seedlings grown in Pi-sufficient conditions were transferred to either No Phosphate medium (NP) or Low Phosphate medium (LP, $25 \mu \mathrm{M} \mathrm{Pi}$ ) containing 20 or $30 \mu \mathrm{M}$ uranyl nitrate (U20, U30) for 1 or 3 days. A- Staining of callose using aniline blue. Aniline blue fluorescence was observed using an epifluorescence microscope under a DAPI filter. Time of exposure was set at $200 \mathrm{~ms}$. B- Staining of lignin using the Wiesner solution. Lignin staining was observed with an optical microscope under bright field. Observations were made at magnification $\times 200$. Images are representative of $n=5$ to 10 samples analyzed at each time-point in two to four independent experiments. Scale bar $=100 \mu \mathrm{m}$.

Lignin is a complex polymer of the cell wall that mainly accumulates in root tissues at the xylem vessels and the endodermis. Lignin contains many functional groups (e.g. hydroxyl, carboxyl) that can bind essential and toxic divalent metal ions and control their transfer between the apoplasm and the symplasm (reviewed in Liu et al., 2018). We analyzed the effect of $U$ on lignin deposition in roots using the Wiesner staining procedure. While no lignin staining was observed in LP, NP and U20 conditions, the accumulation of the polymer was detected in the stele of the elongation zone after 24 hours of U30 treatment (Fig. 8B). At day 3 in U30, lignin staining was even stronger and covered all cell files of the elongation zone except the epidermis and the cortex (Fig. 8B). Together these data suggested that lignin accumulation in the root tip has a role to address the stress induced by toxic doses of $U$. 


\section{DISCUSSION}

In this study, we deciphered the physiological and cellular consequences of $U$ stress on the root system of Arabidopsis seedlings. Since both root development and $U$ bioavailability are influenced by many environmental cues, including the physicochemical properties and mineral composition of the rhizosphere, we set up experimental conditions to specifically analyze the effects of $U$. To this aim our hydroponic system comprised a complete mineral nutrient solution in which Pi was reduced from 250 $\mu \mathrm{M}$ (Pi-sufficiency) to $25 \mu \mathrm{M}$ (Pi-limitation) during U stress. This Pi-limiting condition is in the same order of magnitude as Pi bioavailability in soil solution $(10 \mu \mathrm{M})$ (Abel, 2017) and limits the formation of uranyl phosphate complexes that decrease U bioavailability and toxicity (Vanhoudt et al., 2008; Misson et al., 2009; Laurette et al., 2012b; Doustaly et al., 2014; Berthet et al., 2018). In these conditions, viability staining of root apices identified two concentrations of uranyl nitrate inducing a contrasted response to the radionuclide (Fig. 1).

At an initial concentration of $20 \mu \mathrm{M}$ in the medium $\mathrm{U}$ induced significant changes in the root architecture, with an improved primary root growth and a reduction of secondary root formation (Fig. 4). Enhanced primary root growth was correlated with a very strong stimulation of the mitotic activity of the primary root apex, as early as the first day of $U$ stress (Fig. 5). Such a positive effect of a stressor at low dose is called hormesis (for a review, see Poschenrieder et al., 2013). Hormesis has already been described in Arabidopsis plants challenged with U (Misson et al., 2009; Vanhoudt et al., 2011a). It could result from an overcompensation of the early perturbation in homeostasis, e.g. ROS production and induction of stress-induced antioxidants, leading to cellular responses that are beneficial for growth (Poschenrieder et al., 2013). Contamination of the medium with $30 \mu \mathrm{M}$ uranyl nitrate revealed $\mathrm{U}$ toxicity with the arrest of primary root growth since the first day of stress (Fig. 5) and cell death that spread all over the primary apex after three days (Fig. 1). The U30 condition was also characterized by an increased formation of secondary and higher-order lateral roots (Fig. 4).

The changes of root architecture observed in the presence of $U$ were not similar to, but shared features with, the ones found in the NP condition, suggesting a relationship between $U$ toxicity and Pi deficiency. This was confirmed through monitoring Pi content in seedlings. Depletion of the medium with $\mathrm{Pi}$ (NP condition) or contamination of the low Pi medium with $20 \mu \mathrm{M}$ uranyl (U20 condition) triggered a similar Pi deficiency in seedlings (Fig. 2B). In the U30 condition, Pi deficiency was slightly but significantly more important, in agreement with the increased amount of $U$ incorporated into seedlings as compared to U20 (Fig. 2A). Phosphate deficiency was correlated with a depletion of Pi in the medium (Fig. S2), likely due to the formation of U-Pi precipitates, and to the interaction of $\mathrm{U}$ with $\mathrm{Pi}$ homeostasis in the seedlings. Indeed, it has been shown previously by ${ }^{31} \mathrm{P}$ NMR spectroscopy that $\mathrm{U}$ triggers an important depletion of the cytosolic pools of $\mathrm{Pi}$ and phosphorylated metabolites (Berthet et al., 2018).

Recent advances have been made in the understanding of the sensory mechanisms that monitor external Pi and regulate Pi homeostasis during root development (reviewed in Abel, 2017). The different physiological parameters we analyzed in roots challenged with $U$ indicate that part of the radionuclide effects are associated with $\mathrm{Pi}$ deprivation and the complex interaction between $\mathrm{Pi}$ sensing and $\mathrm{Fe}$ homeostasis in root tips. Indeed, both $U$ treatment at a sub-toxic dose (Fig. 3) and Pi deficiency (Müller 
et al., 2015) induced Fe accumulation in the root apex and the transition zone (located between the meristem and the elongation zone). The pattern of Fe distribution was correlated with the sites of production of ROS and callose deposition upon Pi deprivation (Müller et al., 2015). We observed a similar relationship between Fe (Fig. 3), ROS (Fig. 7A) and callose deposition (Fig. 8A) in the root apices of seedlings challenged with U. Cell-type-specific callose deposition was shown to inhibit symplastic communication through plasmodesmata and to modulate the maintenance of the root apical meristem upon Pi deprivation (Müller et al., 2015). Although similar patterns of callose deposition were observed in Pi-deprived (Müller et al., 2015) or U-contaminated (Fig. 8A) conditions, the first leads to meristem arrest and stop of primary root growth, whereas in the second the mitotic activity is highly increased (Fig. 5), resulting in a stimulation of primary root growth (Fig. 4).

In the case of $U$ stress, the adjustment of cell-to-cell communication by callose deposition in the cell wall is likely a way to limit the diffusion of the radionuclide in the root tip, as reported for other toxic trace metals (reviewed in Krzesłowska, 2011). This strategy is probably sufficient per se to limit $U$ spread at moderate doses (e.g. U20 condition). At a higher concentration of $\mathrm{U}$, we observed that a second defense polymer, lignin, accumulated in the stele of root tip (Fig. 8B). Lignin deposition in the cell wall can contribute to an improved capacity of uranyl ion binding through hydroxyl or carboxyl moieties of the polymer. The preferential localization of $U$ deposits in the cell wall of root cells supports this assumption (Misson et al., 2009; Laurette et al., 2012a). Thus, lignin can participate in forming a physical barrier to avoid $U$ propagation to the whole plant, as reported for other trace elements (Lequeux et al., 2010; Liu et al., 2018).

In addition to ROS, NO is likely involved in the signaling of $U$ stress in the root. Indeed, our results showed that ROS and NO both accumulate in the root tip as early as 6 hours of treatment in U30 (Fig. 7). This is in agreement with a previous report showing that NO is produced in the roots of Arabidopsis plants challenged with $U$ and that NO inducers or inhibitors of NO synthesis modulate the toxicity of the radionuclide (Tewari et al., 2015). The role of $\mathrm{NO}$ as a signal molecule during $\mathrm{U}$ stress is also supported by the demonstration that (i) an excess of Fe reduces root tip growth through a NO-mediated mechanism (Zhang et al., 2018), (ii) lignin is synthesized in response to high NO levels (Böhm et al., 2010; Corti Monzón et al., 2014), and (iii) NO influences the cell cycle in a dose-dependent manner, from stimulation to inhibition (Novikova et al., 2017).

As explained above, the physiological and cellular effects of $U$ depend, in part, on a cascade of events related to Pi deprivation signaling (Fe-dependent ROS production and callose deposition) (Fig. 9). The fact that $\mathrm{U}$ stress and $\mathrm{Pi}$ deprivation per se do not result in identical symptoms indicates that the radionuclide has direct toxic effects on cellular components and/or is able to amplify Pi deficiency (Fig. 9). Yet, the first hypothesis is not supported by experimental evidences since no molecular target of the uranyl ion has been identified in plants. However, the amplification of the Pi deprivation signaling cascade is likely to occur through the interaction of $U$ with Fe homeostasis. Indeed, the competition between $\mathrm{U}$ and $\mathrm{Fe}$ for the formation of complexes with $\mathrm{Pi}$ (Berthet et al., 2018) and/or for protein ligands could release $\mathrm{Fe}^{3+}$ and increase the production of ROS via the Fenton reaction (Fig. 9). Ferritin, which control Fe related oxidative stress in plants (Briat et al., 2010; Reyt et al., 2015), is an attractive, but not yet proven, protein candidate that could explain part of the toxicity of $U$ in the root system. Indeed, $U$ 
has been shown to interact in vitro with a human ferritin (Michon et al., 2010) and may actually replace Fe into ferritins in bacteria (Cvetkovic et al., 2010). Ferritin is not the only candidate for the binding of the radionuclide since uranyl has been shown to replace $\mathrm{Fe}^{3+}$ in some proteins, including serum transferrin that is involved in Fe transport (Vidaud et al., 2007). The identification of plant proteins that are able to bind the uranyl ion is a future challenge to gain insight into the molecular mechanisms that mediate the direct toxic effects of $U$.

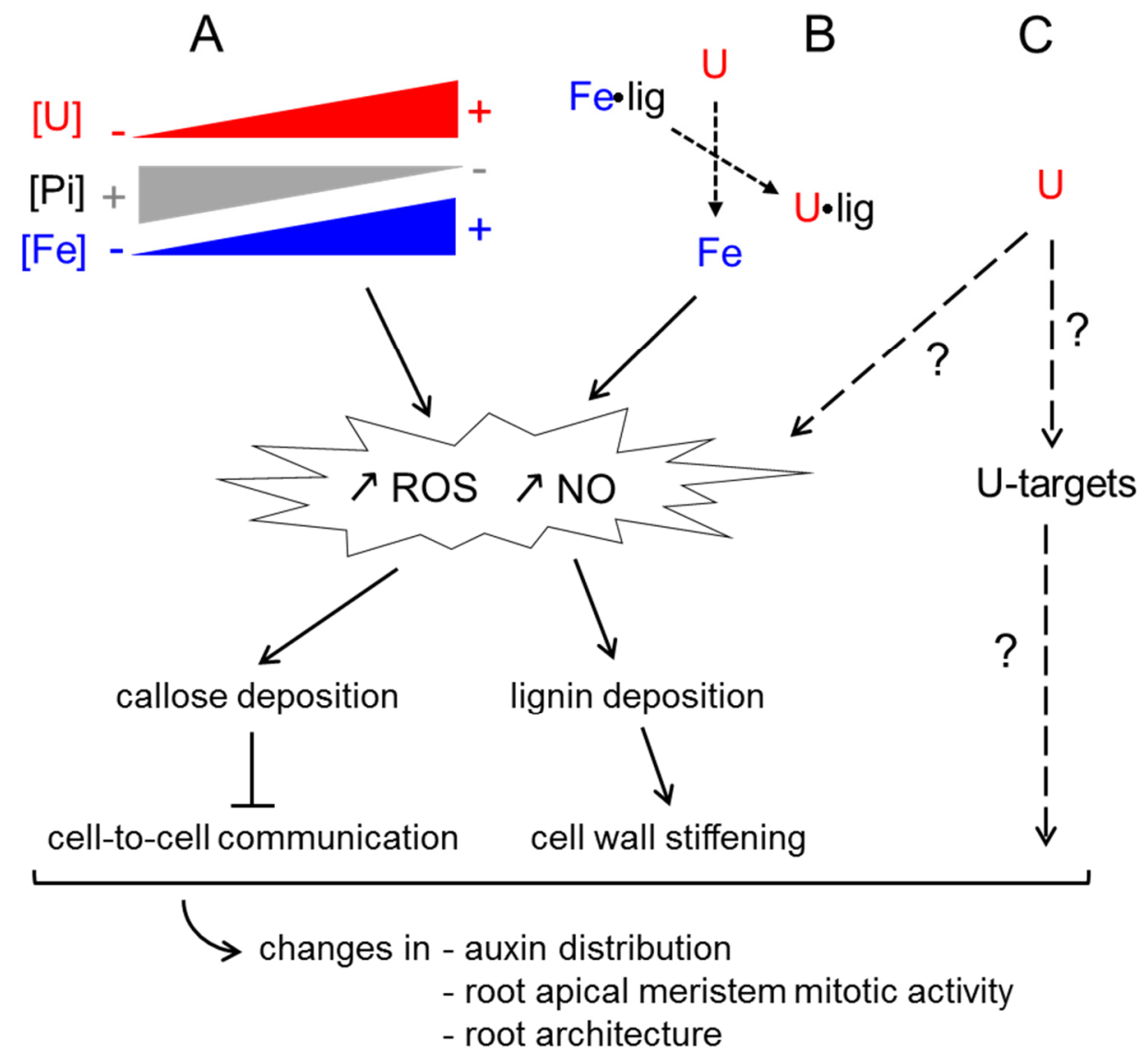

Figure 9. Tentative model showing the physiological and cellular responses of Arabidopsis roots to $U$ stress. This model illustrates the three processes that could contribute to changes in the root architecture of seedlings challenged with $\mathrm{U}$. A- First, $\mathrm{U}$ accumulation induces $\mathrm{Pi}$ deprivation, which in turn triggers Fe redistribution in root tissues, ROS production, and callose deposition. These events are similar to the one described for the signaling cascade of Pi sensing in the root apex (Muller et al., 2015; Abel, 2017). B- Second, the competition between $U$ and Fe for the formation of complexes with diverse ligands $\left(\mathrm{Pi}\right.$, organic acids, cell wall components, proteins...) could release free $\mathrm{Fe}^{3+}$, trigger the production of ROS, and thus amplify the Pi sensing cascade. C- Third, $U$ could directly affect root growth and architecture through toxic effects on specific targets, which are yet not known. NO is, in addition to ROS, a secondary messenger involved in $U$ stress signaling in the root. 


\section{ACKNOWLEDGEMENTS}

We thank Dr. Christian Hermans (laboratory of Plant Physiology and Molecular Genetics, Université Libre de Bruxelles, Brussels, Belgium) for providing us with seeds of the Arabidopsis lines DR5::GFP and CYCB1::GUS, and Dr. Antoine Larrieu (laboratoire Reproduction et Développement des Plantes, , Lyon, France) for seeds of PIN2::PIN2-GFP.

The PhD fellowship to NBCS was funded by the Région Auvergne Rhône-Alpes. This work was funded by a grant from the Toxicology program of the Commissariat à l'Energie Atomique et aux Energies Alternatives, the French National Research Agency for the GreenU project (ANR-17-CE34-0007) and the LabEx GRAL (ANR-10-LABX-49-01).

\section{CONTRIBUTIONS}

NBCS, CA, JB, SR conceived and designed the study; NBCS, SR performed the experiments; NBCS, CA, JB, SR analyzed the data; NBCS, JB, SR wrote the paper. JB and SR should be considered joint senior author. 


\section{REFERENCES}

Abel, S., 2017. Phosphate scouting by root tips. Current Opinion in Plant Biology 39, 168-177.

Anke, M., Seeber, O., Mueller, R., Schaefer, U., Zerull, J., 2009. Uranium transfer in the food chain from soil to plants, animals and man. Chemie Der Erde-Geochemistry 69, 75-90.

Aranjuelo, I., Doustaly, F., Cela, J., Porcel, R., Mueller, M., Aroca, R., Munne-Bosch, S., Bourguignon, J., 2014. Glutathione and transpiration as key factors conditioning oxidative stress in Arabidopsis thaliana exposed to uranium. Planta 239, 817-830.

Berthet, S., Villiers, F., Alban, C., Serre, N.B.C., Martin-Laffon, J., Figuet, S., Boisson, A.M., Bligny, R., Kuntz, M., Finazzi, G., Ravanel, S., Bourguignon, J., 2018. Arabidopsis thaliana plants challenged with uranium reveal new insights into iron and phosphate homeostasis. New Phytologist 217, 657-670.

Blilou, I., Xu, J., Wildwater, M., Willemsen, V., Paponov, I., Friml, J., Heidstra, R., Aida, M., Palme, K., Scheres, B., 2005. The PIN auxin efflux facilitator network controls growth and patterning in Arabidopsis roots. Nature 433, 39-44.

Böhm, F.M.L.Z., Ferrarese, M.d.L.L., Zanardo, D.I.L., Magalhaes, J.R., Ferrarese-Filho, O., 2010. Nitric oxide affecting root growth, lignification and related enzymes in soybean seedlings. Acta Physiologiae Plantarum 32, 1039-1046.

Briat, J.-F., Ravet, K., Arnaud, N., Duc, C., Boucherez, J., Touraine, B., Cellier, F., Gaymard, F., 2010. New insights into ferritin synthesis and function highlight a link between iron homeostasis and oxidative stress in plants. Annals of Botany 105, 811-822.

Corpas, F., Barroso, J., 2015. Functions of Nitric Oxide (NO) in Roots during Development and under Adverse Stress Conditions. Plants 4, 240-252.

Correa-Aragunde, N., Graziano, M., Lamattina, L., 2004. Nitric oxide plays a central role in determining lateral root development in tomato. Planta 218, 900-905.

Corti Monzón, G., Pinedo, M., Di Rienzo, J., Novo-Uzal, E., Pomar, F., Lamattina, L., de la Canal, L., 2014. Nitric oxide is required for determining root architecture and lignin composition in sunflower. Supporting evidence from microarray analyses. Nitric Oxide 39, 20-28.

Cvetkovic, A., Menon, A.L., Thorgersen, M.P., Scott, J.W., Poole li, F.L., Jenney Jr, F.E., Lancaster, W.A., Praissman, J.L., Shanmukh, S., Vaccaro, B.J., Trauger, S.A., Kalisiak, E., Apon, J.V., Siuzdak, G., Yannone, S.M., Tainer, J.A., Adams, M.W.W., 2010. Microbial metalloproteomes are largely uncharacterized. Nature 466, 779-782.

De Smet, I., Tetsumura, T., De Rybel, B., Frey, N.F.d., Laplaze, L., Casimiro, I., Swarup, R., Naudts, M., Vanneste, S., Audenaert, D., Inze, D., Bennett, M.J., Beeckman, T., 2007. Auxin-dependent regulation of lateral root positioning in the basal meristem of Arabidopsis. Development 134, 681-690.

Doonan, J., 1996. Plant growth: Roots in the cell cycle. Current Biology 6, 788-789.

Doustaly, F., Combes, C., Fiévet, J., Berthet, S., Hugouvieux, V., Bastien, O., Aranjuelo, I., Leonhardt, N., Rivasseau, C., Carrière, M., Vavasseur, A., Renou, J.P., Vandenbrouck, Y., Bourguignon, J., 2014. Uranium perturbs signaling and iron uptake response in Arabidopsis thaliana roots. Metallomics 6, 809--821.

Ebbs, S.D., Norvell, W.A., Kochian, L.V., 1998. The effect of acidification and chelating agents on the solubilization of uranium from contaminated soil. Journal of Environmental Quality 27, 1486-1494.

Eleftheriou, E.P., Adamakis, I.D., Panteris, E., Fatsiou, M., 2015. Chromium-Induced Ultrastructural Changes and Oxidative Stress in Roots of Arabidopsis thaliana. International Journal of Molecular Sciences 16, 15852-15871.

Howden, R., Cobbett, C.S., 1992. Cadmium-Sensitive Mutants of Arabidopsis thaliana. Plant Physiology 100, 100-107.

Hugouvieux, V., Dutilleul, C., Jourdain, A., Reynaud, F., Lopez, V., Bourguignon, J., 2009. Arabidopsis putative selenium-binding protein1 expression is tightly linked to cellular sulfur demand and can reduce sensitivity to stresses requiring glutathione for tolerance. Plant Physiology 151, 768-781.

Jain, A., Poling, M.D., Smith, A.P., Nagarajan, V.K., Lahner, B., Meagher, R.B., Raghothama, K.G., 2009. Variations in the Composition of Gelling Agents Affect Morphophysiological and Molecular Responses to Deficiencies of Phosphate and Other Nutrients. Plant Physiology 150, 1033-1049.

Jones, K., Kim, D.W., Park, J.S., Khang, C.H., 2016. Live-cell fluorescence imaging to investigate the dynamics of plant cell death during infection by the rice blast fungus Magnaporthe oryzae. BMC Plant Biol 16, 69.

Kanno, S., Cuyas, L., Javot, H., Bligny, R., Gout, E., Dartevelle, T., Hanchi, M., Nakanishi, T.M., Thibaud, M.-C., Nussaume, L., 2016. Performance and Limitations of Phosphate Quantification: Guidelines for Plant Biologists. Plant and Cell Physiology 57, 690-706.

Kazan, K., 2013. Auxin and the integration of environmental signals into plant root development. Annals of Botany 112, 1655-1665. 
Konietschke, F., Placzek, M., Schaarschmidt, F., Hothorn, L.A., 2015. nparcomp: An R Software Package for Nonparametric Multiple Comparisons and Simultaneous Confidence Intervals. Journal of Statistical Software 64, 1-17.

Kopittke, P.M., Moore, K.L., Lombi, E., Gianoncelli, A., Ferguson, B.J., Blamey, F.P., Menzies, N.W., Nicholson, T.M., McKenna, B.A., Wang, P., Gresshoff, P.M., Kourousias, G., Webb, R.I., Green, K., Tollenaere, A., 2015. Identification of the primary lesion of toxic aluminum in plant roots. Plant Physiology 167, 1402-1411.

Krzesłowska, M., 2011. The cell wall in plant cell response to trace metals: polysaccharide remodeling and its role in defense strategy. Acta Physiologiae Plantarum 33, 35-51.

Laskowski, M., Ten Tusscher, K.H., 2017. Periodic Lateral Root Priming: What Makes It Tick? Plant Cell 29, 432-444.

Laurette, J., Larue, C., Llorens, I., Jaillard, D., Jouneau, P.-H., Bourguignon, J., Carrière, M., 2012a. Speciation of uranium in plants upon root accumulation and root-to-shoot translocation: A XAS and TEM study. Environmental and Experimental Botany 77, 87-95.

Laurette, J., Larue, C., Mariet, C., Brisset, F., Khodja, H., Bourguignon, J., Carrière, M., 2012b. Influence of uranium speciation on its accumulation and translocation in three plant species: Oilseed rape, sunflower and wheat. Environmental and Experimental Botany 77, 96-107.

Lequeux, H., Hermans, C., Lutts, S., Verbruggen, N., 2010. Response to copper excess in Arabidopsis thaliana: Impact on the root system architecture, hormone distribution, lignin accumulation and mineral profile. Plant Physiology and Biochemistry 48, 673-682.

Liu, Q., Luo, L., Zheng, L., 2018. Lignins: Biosynthesis and Biological Functions in Plants. International Journal of Molecular Sciences 19, 1-16.

Locato, V., Paradiso, A., Sabetta, W., De Gara, L., de Pinto, M.C., 2016. Nitric Oxide and Reactive Oxygen Species in PCD Signaling, Advances in Botanical Research. Elsevier, pp 165-192.

Meyer, C.L., Juraniec, M., Huguet, S., Chaves-Rodriguez, E., Salis, P., Isaure, M.P., Goormaghtigh, E., Verbruggen, N., 2015. Intraspecific variability of cadmium tolerance and accumulation, and cadmiuminduced cell wall modifications in the metal hyperaccumulator Arabidopsis halleri. Journal of Experimental Botany 66, 3215-3227.

Michon, J., Frelon, S., Garnier, C., Coppin, F., 2010. Determinations of Uranium(VI) Binding Properties with some Metalloproteins (Transferrin, Albumin, Metallothionein and Ferritin) by Fluorescence Quenching. Journal of Fluorescence 20, 581-590.

Misson, J., Henner, P., Morello, M., Floriani, M., Wu, T.D., Guerquin-Kern, J.L., Fevrier, L., 2009. Use of phosphate to avoid uranium toxicity in Arabidopsis thaliana leads to alterations of morphological and physiological responses regulated by phosphate availability. Environmental and Experimental Botany 67, 353-362.

Mittler, R., 2017. ROS Are Good. Trends in Plant Science 22, 11-19.

Müller, J., Toev, T., Heisters, M., Teller, J., Moore, Katie L., Hause, G., Dinesh, Dhurvas C., Bürstenbinder, K., Abel, S., 2015. Iron-Dependent Callose Deposition Adjusts Root Meristem Maintenance to Phosphate Availability. Developmental Cell 33, 216-230.

Nedukha, O.M., 2015. Callose: Localization, functions, and synthesis in plant cells. Cytology and Genetics 49, 49-57.

Novikova, G.V., Mur, L.A., Nosov, A.V., Fomenkov, A.A., Mironov, K.S., Mamaeva, A.S., Shilov, E.S., Rakitin, V.Y., Hall, M.A., 2017. Nitric Oxide Has a Concentration-Dependent Effect on the Cell Cycle Acting via EIN2 in Arabidopsis thaliana Cultured Cells. Frontiers in Physiology 8, 1-11.

Pearson, R.G., 1963. Hard and soft acids and bases. Journal of the American Chemical Society 85, 3533-3539.

Péret, B., Clément, M., Nussaume, L., Desnos, T., 2011. Root developmental adaptation to phosphate starvation: better safe than sorry. Trends in Plant Science 16, 442-450.

Petrasek, J., Friml, J., 2009. Auxin transport routes in plant development. Development 136, 2675-2688.

Poschenrieder, C., Cabot, C., Martos, S., Gallego, B., Barceló, J., 2013. Do toxic ions induce hormesis in plants? Plant Science 212, 15-25.

Potters, G., Pasternak, T.P., Guisez, Y., Jansen, M.A.K., 2009. Different stresses, similar morphogenic responses: integrating a plethora of pathways. Plant Cell and Environment 32, 158-169.

Pradhan Mitra, P., Loqué, D., 2014. Histochemical Staining of Arabidopsis thaliana Secondary Cell Wall Elements. Journal of Visualized Experiments, e51381.

Reyt, G., Boudouf, S., Boucherez, J., Gaymard, F., Briat, J.-F., 2015. Iron- and Ferritin-Dependent Reactive Oxygen Species Distribution: Impact on Arabidopsis Root System Architecture. Molecular Plant 8, 439-453.

Roschzttardtz, H., Conejero, G., Curie, C., Mari, S., 2009. Identification of the Endodermal Vacuole as the Iron Storage Compartment in the Arabidopsis Embryo. Plant Physiology 151, 1329-1338. 
Saenen, E., Horemans, N., Vanhoudt, N., Vandenhove, H., Biermans, G., Van Hees, M., Wannijn, J., Vangronsveld, J., Cuypers, A., 2015. Induction of Oxidative Stress and Antioxidative Mechanisms in Arabidopsis thaliana after Uranium Exposure at $\mathrm{pH}$ 7.5. International Journal of Molecular Sciences $16,12405-12423$.

Saenen, E., Horemans, N., Vanhoudt, N., Varidenhove, H., Biermans, G., Van Hees, M., Wannijn, J., Vangronsveld, J., Cuypers, A., 2014. The $\mathrm{pH}$ strongly influences the uranium-induced effects on the photosynthetic apparatus of Arabidopsis thaliana plants. Plant Physiology and Biochemistry 82, 254261.

Schenk, S.T., Schikora, A., 2016. Staining of Callose Deposition in Root and Leaf Tissues. Bio-protocol 5, e1429.

Schnug, E., Lottermoser, B.G., 2013. Fertilizer-Derived Uranium and its Threat to Human Health. Environmental Science \& Technology 47, 2433-2434.

Svistoonoff, S., Creff, A., Reymond, M., Sigoillot-Claude, C., Ricaud, L., Blanchet, A., Nussaume, L., Desnos, T., 2007. Root tip contact with low-phosphate media reprograms plant root architecture. Nature Genetics 39, 792-796.

Team, R.C., 2017. R: A Language and Environment for Statistical Computing, 3.3.2 ed. R Foundation for Statistical Computing, Vienna, Austria.

Tewari, R., Horemans, N., Nauts, R., Wannijn, J., Van Hees, M., Vandenhove, H., 2015. Uranium exposure induces nitric oxide and hydrogen peroxide generation in Arabidopsis thaliana. Environmental and Experimental Botany 120, 55-64.

Thiebault, C., Carriere, M., Milgram, S., Simon, A., Avoscan, L., Gouget, B., 2007. Uranium Induces Apoptosis and Is Genotoxic to Normal Rat Kidney (NRK-52E) Proximal Cells. Toxicological Sciences 98, 479-487.

Vandenhove, H., 2002. European sites contaminated by residues from the ore-extracting andprocessing industries. International Congress Series 1225, 307-315.

Vanhoudt, N., Cuypers, A., Horemans, N., Remans, T., Opdenakker, K., Smeets, K., Bello, D.M., Havaux, M., Wannijn, J., Van Hees, M., Vangronsveld, J., Vandenhove, H., 2011c. Unraveling uranium induced oxidative stress related responses in Arabidopsis thaliana seedlings. Part II: responses in the leaves and general conclusions. Journal of Environmental Radioactivity 102, 638-645.

Vanhoudt, N., Horemans, N., Biermans, G., Saenen, E., Wannijn, J., Nauts, R., Van Hees, M., Vandenhove, H., 2014. Uranium affects photosynthetic parameters in Arabidopsis thaliana. Environmental and Experimental Botany 97, 22-29.

Vanhoudt, N., Vandenhove, H., Horemans, N., Bello, D.M., Van Hees, M., Wannijn, J., Carleer, R., Vangronsveld, J., Cuypers, A., 2011a. Uranium induced effects on development and mineral nutrition of Arabidopsis thaliana. Journal of Plant Nutrition 34, 1940-1956.

Vanhoudt, N., Vandenhove, H., Horemans, N., Remans, T., Opdenakker, K., Smeets, K., Bello, D.M., Wannijn, J., Van Hees, M., Vangronsveld, J., Cuypers, A., 2011b. Unraveling uranium induced oxidative stress related responses in Arabidopsis thaliana seedlings. Part I: responses in the roots. Journal of Environmental Radioactivity 102, 630-637.

Vanhoudt, N., Vandenhove, H., Smeets, K., Remans, T., Van Hees, M., Wannijn, J., Vangronsveld, J., Cuypers, A., 2008. Effects of uranium and phosphate concentrations on oxidative stress related responses induced in Arabidopsis thaliana. Plant Physiology and Biochemistry 46, 987-996.

Vidaud, C., Bourgeois, D., Meyer, D., 2012. Bone as Target Organ for Metals: The Case of f-Elements. Chemical Research in Toxicology 25, 1161-1175.

Vidaud, C., Gourion-Arsiquaud, S., Rollin-Genetet, F., Torne-Celer, C., Plantevin, S., Pible, O., Berthomieu, C., Quemeneur, E., 2007. Structural consequences of binding of UO2(2+) to apotransferrin: can this protein account for entry of uranium into human cells? Biochemistry 46, 22152226.

Zhang, L., Li, G., Wang, M., Di, D., Sun, L., Kronzucker, H.J., Shi, W., 2018. Excess iron stress reduces root tip zone growth through nitric oxide-mediated repression of potassium homeostasis in Arabidopsis. New Phytologist 219, 259-274. 


\section{Supporting information}

Uncovering the physiological and cellular effects of uranium on the root system of Arabidopsis thaliana

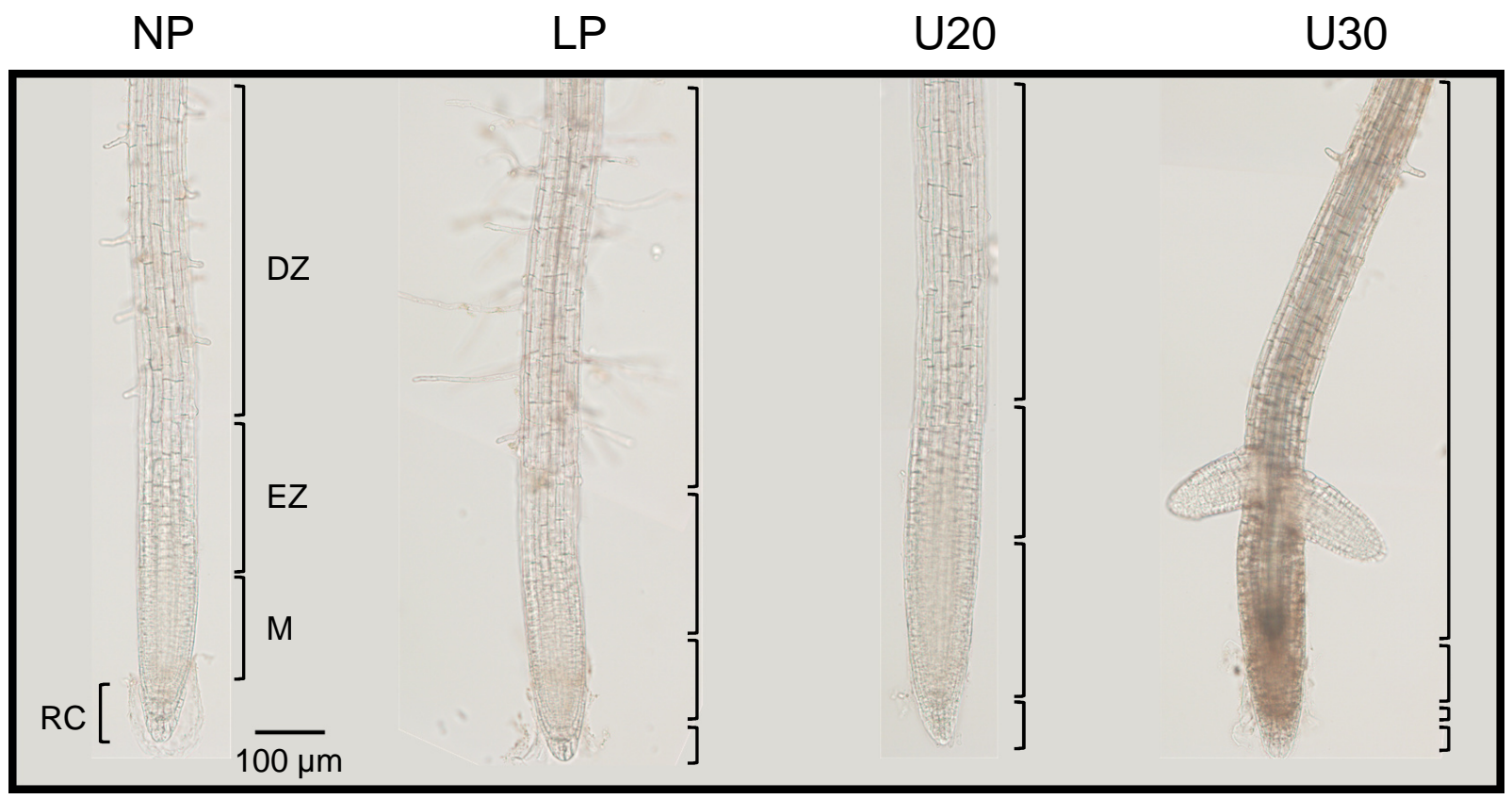

Figure S1. Structure of the Arabidopsis root apex during $U$ stress. The organization of the different zones of the root apex in our experimental conditions is shown. RC, root cap; M, meristem; EZ, elongation zone; DZ, differentiation zone. Observations were made using an optical microscope under bright light (magnification $\times 200$ ). Scale bar $=100 \mu \mathrm{m}$. 
A

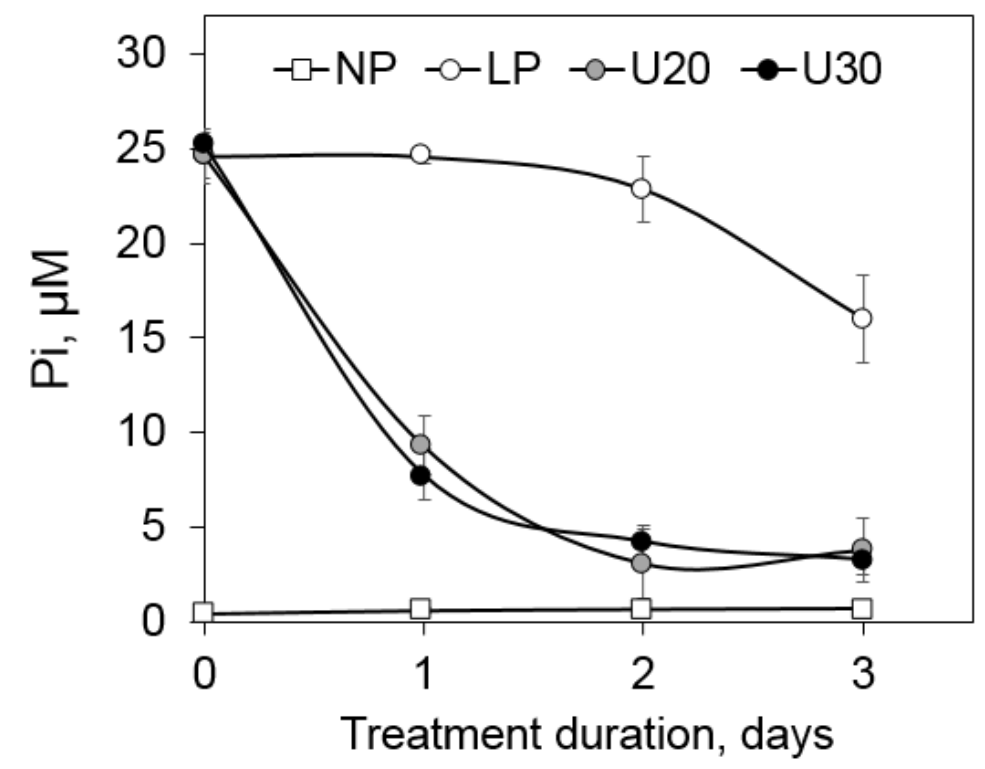

B

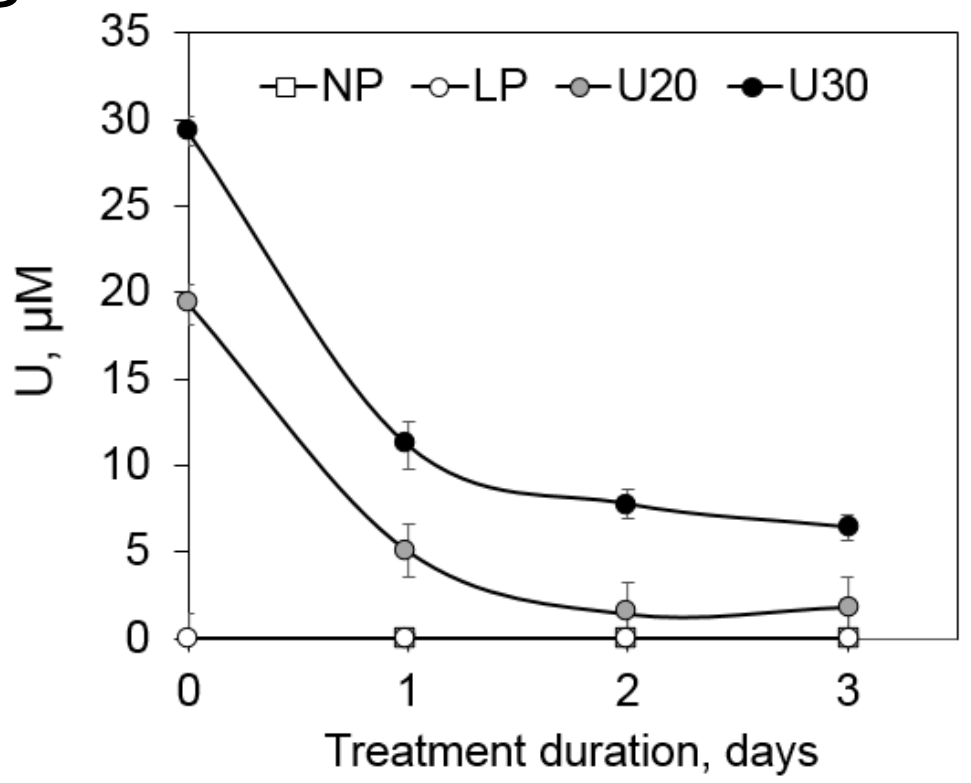

Figure S2. Phosphate and uranium availability in the root absorption zone of the culture media. Aliquots of the culture media were collected in the root absorption zone up to three days after the transfer of Col-0 seedlings to either No Phosphate medium (NP) or Low Phosphate medium (LP, $25 \mu \mathrm{M}$ Pi) containing 20 or $30 \mu \mathrm{M}$ uranyl nitrate (U20, U30). A- The amount of soluble Pi was measured spectrophotometrically using the molybdate/malachite green reagents. B- The amount of $U$ was determined by ICP-MS. Curves represent mean \pm SD with $n=6$ to 16 samples per condition. 